\title{
THE DIFFERENTIABLE SPACE STRUCTURES OF MILNOR CLASSIFYING SPACES, SIMPLICIAL COMPLEXES, AND GEOMETRIC REALIZATIONS
}

\author{
MARK A. MOSTOW
}

In this paper we develop a simple and very general concept, called a differentiable space, by means of which one can define smooth functions, differential forms, and de Rham cohomology on a wide variety of topological spaces without making use of any concept of tangent vectors. Our notion of differentiable space is related to those of J. W. Smith [30] and K. T. Chen [8], [9] but differs in some important respects. We prove some theorems which give sufficient conditions for the de Rham cohomology defined in this way to equal the real singular cohomology of a space. In particular, we show that simplicial complexes, Milnor classifying spaces $B G$ of Lie groups $G$, and geometric realizations of semi-simplicial manifolds have natural differentiable space structures which yield their correct real cohomology. Unlike J. Dupont's [14] and C. Watkiss' [37] definitions of differential forms on geometric realizations based on their "piecewise smooth" structure, the differentiable space approach permits one to define smooth morphisms from a manifold $M$ to a non-manifold like Milnor's $B G$. For example, the classifying map $f: M \rightarrow B G$ of a $G$-bundle on $M$ with smooth transition functions is a (smooth) morphism of differentiable spaces, provided that $f$ is constructed using a smooth partition of unity on $M$. Thus not only can one construct explicit characteristic forms on $B G$ (by using a universal connection form on $E G)$, but one can also pull them back at the form level to the de Rham complex of $M$ via $f^{*}: A^{*}(B G) \rightarrow A^{*}(M)$. In this way one gets a somewhat different perspective on the Chern-Weil homomorphism which combines topology (classifying spaces) and geometry (connections and curvatures).

A differentiable space is defined to be simply a topological space $X$ together with a sheaf $\mathbf{C}^{\infty} X$ of germs of continuous real-valued functions on $X$, called smooth functions, satisfying the closure condition

If $f_{1}, \cdots, f_{n}$ are smooth functions on $X$, and $g$ is a smooth function on $\mathbf{R}^{n}$, then $g\left(f_{1}, \cdots, f_{n}\right)$ is a smooth function on $X$.

Communicated by R. S. Palais, May 6, 1977. Part of this work was done while the author was on NSF Grant MCS 76-06562. 
In other words, one simply takes some collection of functions on $X$ and decrees them to be smooth. For example, on a simplicial complex it is natural to take the barycentric coordinate functions, as well as functions which locally are smooth functions of finitely many barycentric coordinates, to be the smooth functions.

Differential forms on a differentiable space $X$ are defined to be abstract symbols $\eta=\Sigma f_{i_{0}} d f_{i_{1}} \wedge \cdots \wedge d f_{i_{n}}$ (locally finite sum), where the $f_{i_{1}}$ are smooth functions on $X$, modulo the equivalence relation defined by calling two such symbols $\eta_{1}$ and $\eta_{2}$ equivalent if each smooth map $\phi: E \rightarrow X$ ( $E$ open in some $\mathbf{R}^{k}$ ) pulls back $\eta_{1}$ and $\eta_{2}$ to identical forms on $E$.

The contents of this paper are as follows.

$\$ 1$ defines differentiable spaces and their morphisms and presents a number of examples, including smooth manifolds, classifying spaces of Lie groupoids, and simplicial complexes.

$\$ 2$ defines the de Rham complex of a differentiable space and proves some of its elementary properties, such as the Chain Rule. De Rham cohomology is defined, as usual, as the cohomology of the de Rham complex.

$\$ 3$ defines smooth homotopies and shows that de Rham cohomology is invariant under smooth homotopies in the category of differentiable spaces.

$\$ 4$ compares our definition of differentiable space with those of $\mathrm{J}$. W. Smith and K. T. Chen, which are related to ours but not equivalent. The approach of Whitney, Sullivan, Thom, et al., who defined differential forms on simplicial complexes to be compatible collections of forms on the simplices, is discussed, as is the similar approach used by $\mathrm{C}$. Watkiss and $\mathrm{J}$. Dupont to define differential forms on geometric realizations of semi-simplicial manifolds. We also discuss the de Rham double complex of a semi-simplicial space, as defined by Bott, Shulman, and Stasheff, the Weil algebra, and other related ideas.

In $\$ 5$ we prove two theorems which give sufficient conditions for de Rham cohomology to equal real singular cohomology. The first of these (Theorem 5.2) says that if $X$ is paracompact, admits smooth partitions of unity subordinate to any open cover, and is locally smoothly contractible, then $H_{D R}^{*}(X)=H_{\text {sing }}^{*}(X ; \mathbf{R})$. After discussing the Čech-singular and Čech-de Rham double complexes of a differentiable space we prove (Theorem 5.5) that if a de Rham isomorphism holds on every finite intersection of some open cover of $X$ which admits a smooth partition of unity, then $H_{D R}^{*}(X)=$ $H_{\text {sing }}^{*}(X)$.

$\$ 6$ discusses the problem of when an open cover of a differentiable space admits a smooth partition of unity. This question is important if we want to apply the de Rham theorems mentioned above. The results of this section 
follow from a recent theorem of $\mathrm{H}$. Torunczyk, who in the course of studying smooth partitions of unity on Banach spaces gave what amount to necessary and sufficient conditions for a differentiable space whose underlying topological space is metrizable to admit smooth partitions of unity (subordinate to any open cover). An easy consequence of his theorem is that any simplicial complex in the metric topology admits smooth partitions of unity.

In $\$ 7$ we prove that $H_{D R}^{*}(X)=H_{\text {sing }}^{*}(X)$ for any simplicial complex $X$ in either the weak or the metric (strong) topology. We show that our de Rham complexes $A^{*}\left(X_{w}\right)$ and $A^{*}\left(X_{s}\right)$ (arising from the weak and strong topologies on $X$ ) can be regarded as subcomplexes of the complex $A_{c}^{*}(X)$ of compatible forms on the simplices of $X$. (We shall call $A_{c}^{*}(X)$ "Whitney's complex", though it differs from Whitney's construction [40] in using smooth forms instead of flat cochains.) When $X$ is a finite or locally finite simplicial complex, all three complexes coincide, but otherwise they differ. Thus one can think of $A^{*}(X)$ as providing an alternate description of Whitney's complex when $X$ is locally finite.

In $\S 8$ we discuss the differentiable space structures and de Rham cohomologies of classifying spaces, and more generally of geometric realizations of a semi-simplicial manifold or differentiable space. This is most easily done if we choose the Milnor-Buffet-Lor classifying space functor $B G$ and the unwound geometric realization $\mu(X)$, because on these spaces there are defined global barycentric coordinates $t_{0}, t_{1}, \cdots$ and other functions $g_{i j}$ or $j_{\sigma}$ (defined on open subsets of $B G$ or $\mu(X)$ ) which can be chosen to be our smooth functions. We show that if $X$ is a semi-simplicial differentiable space and if $H_{D R}^{*}\left(X_{n}\right)=H_{\text {sing }}^{*}\left(X_{n}\right)$ for each $n$, then $H_{D R}^{*}(\mu(X))=H_{\text {sing }}^{*}(\mu(X))$.

$\$ 9$ gives a detailed comparison of six different de Rham complexes defined on geometric realizations, including the double complex of Bott-ShulmanStasheff, the Dupont-Watkiss complex $A^{*}(\|X\|)$ of compatible forms, the unwound versions of these two complexes, and our complexes $A^{*}\left(\mu_{w}(X)\right)$ and $A^{*}\left(\mu_{s}(X)\right)$ defined using the weak or the strong topology on the unwound geometric realization $\mu(X)$. It is shown that all six complexes are chain homotopy equivalent but not isomorphic. The problem of pulling back forms from geometric realizations to manifolds is discussed.

$\$ 10$ presents applications of differentiable spaces. One application, mentioned already, is the presentation of characteristic differential forms for $G$-bundles by explicit universal formulas involving only the transition functions and a smooth partition of unity. Another application is the extension of smooth and $C^{k}$ cohomology theories for spaces with two topologies (studied by the author in [23] and [24]) to non-manifolds like $\left(B \Gamma_{q} \rightarrow B J_{q}\right)$, the Haefliger classifying space for foliations in its sheaf and jet topologies [2]. The 
$C^{k}$ cohomology of $\left(B \Gamma_{q} \rightarrow B J_{q}\right)$ gives rise to characteristic classes of foliations which vary in a $C^{k}$ manner when the foliation is varied smoothly. The latter application was outlined in [24], modulo certain details about differentiable spaces which appear in this paper.

The differentiable space approach is very general and can potentially be applied to many spaces other than those discussed in this paper. In future papers the author plans to present some of these other applications.

Remarks on notation. Smooth always means $C^{\infty}$. Manifolds are locally $C^{\infty}$-diffeomorphic to $\mathbf{R}^{n}(n<\infty)$ but need not be Hausdorff or second countable. The symbol $\mathbf{N}$ denotes the set of nonnegative integers. If $X$ is a semi-simplicial space, then its $n$th space is denoted either $X_{n}$ or $X[n]$.

\section{Definition and examples of differentiable spaces}

Definition. A differentiable space is a topological space $X$ together with, for each open $U$ in $X$, a collection $C^{\infty}(U)$ of continuous real-valued functions on $U$, satisfying the closure conditions:

(i) The rule $U \rightarrow C^{\infty}(U)$ defines a sheaf on $X\left(\right.$ denoted $\left.\mathbf{C}^{\infty} X\right)$.

(ii) For any $n$, if $f_{1}, \cdots, f_{n} \in C^{\infty}(U)$ and $g \in C^{\infty}\left(\mathbf{R}^{n}\right)$ (with the usual meaning), then $g\left(f_{1}, \cdots, f_{n}\right) \in C^{\infty}(U)$.

The elements of $C^{\infty}(X)$ are called smooth functions on $X$.

Remark. If one allows $g \in C^{\infty}(V)\left(V\right.$ open in $\left.\mathbf{R}^{n}\right)$, one gets an equivalent definition.

A basic way to define a differentiable space structure is the following. Let $X$ be a topological space, and let $\left\{f_{a}: U_{a} \rightarrow M_{a}\right\}$ be a collection of continuous functions from open subsets $U_{a}$ covering $X$ to manifolds $M_{a}$. A function $f$ : $U \rightarrow \mathbf{R}(U$ open in $X$ ) is said to be locally a smooth function of finitely many of the $f_{a}$ if for each $x \in U$ there exist a neighborhood $W$ of $x$ in $U$, a finite set of indices $a_{1}, \cdots, a_{n}$, and a smooth map $g: V \rightarrow \mathbf{R}$ (where $V$ is open in $\left.M_{a_{1}} \times M_{a_{2}} \times \cdots \times M_{a_{n}}\right)$ such that for each $i=1, \cdots, n$,

(1) $f_{a_{i}}$ is defined on all of $W$ (i.e., $U_{a_{i}} \supset W$ ),

(2) $f \mid W=g \circ\left(f_{a_{1}}, \cdots, f_{a_{n}}\right)$.

Let $C^{\infty}(U)$ be the set of all such $f$. Then $\left\{X,\left\{C^{\infty}(U)\right\}\right\}$ defines a differentiable space structure on $X$, which we say is generated by $\left\{f_{a}\right\}$.

\section{Examples.}

1. A smooth manifold $M$ with its usual collection of (locally defined) smooth functions is a differentiable space.

2. A topological space $X$ becomes a differentiable space in a trivial way if we decree every continuous function on $X$ to be smooth, i.e., $C^{\infty}(U)=$ (def.) $C(U)$. 
3. Let $\Gamma$ be a Lie groupoid = smooth category with inverses [1] (for example, $\Gamma$ a Lie group $G, \Gamma=$ Haefliger's $\Gamma_{q}$ ). Then $\Gamma$ is a differentiable space, and so is its Milnor-Buffet-Lor geometric realization $B \Gamma$ [5], as we now show. Recall that a point of $B \Gamma$ is specified by a collection $\left\{t_{i}, g_{i j}\right\}$ satisfying

(a) $t_{i} \geqslant 0, i \in \mathbf{N}$, and $t_{i}=0$ for all but a finite number of $i$.

(b) $\Sigma_{i} t_{i}=1$.

(c) $g_{i j} \in \Gamma$, but is defined only on $\left\{t_{i} t_{j} \neq 0\right\}$ (def.) $=U_{i j} \subset B \Gamma$. One endows $B \Gamma$ with either a weak or a strong topology (see $\$ 8$ below). In either topology, we can define a differentiable space structure on $B \Gamma$ by defining, for each open $U$ in $B \Gamma$,

$$
\begin{aligned}
& C^{\infty}(U)=\{f ; U \rightarrow \mathbf{R} \mid f \text { is locally (in the chosen topology) a } \\
& \text { smooth function of finitely many of the functions } g_{i j}: U_{i j} \rightarrow G \\
& \text { and } \left.t_{i}: B \Gamma \rightarrow \mathbf{R}\right\} .
\end{aligned}
$$

4. The constructions of Example 3 work even for infinite-dimensional Lie groupoids like $J_{q}=\left\{\infty\right.$-jets of local diffeomorphisms of $\mathbf{R}^{q}$, with the $C^{\infty}$ topology . We decree the functions $x^{i}$ (coordinates of source), $y^{i}$ (coordinates of target), and $y_{\alpha}^{i}\left(\alpha=\left(\alpha_{1}, \cdots, \alpha_{n}\right), y_{\alpha}^{i}=\alpha\right.$-th partial derivative of $\left.y^{i}\right)$ to be smooth functions on $J_{q}$; also, any function which is locally a smooth function of finitely many of these is called smooth. A function on an open set of $B J_{q}$ is smooth if it is locally a smooth function of finitely many functions of the form $t_{i}$ or $f \circ g_{i j}$, where $f$ is a smooth function on $J_{q}$, and $g_{i j}: U_{i j} \rightarrow J_{q}$ is as in Example 3.

5. A simplicial complex $X$ with either the weak or the strong topology (see $\$ 7$ below) becomes a differentiable space if every function on $X$ which is locally a smooth function of finitely many barycentric coordinates is called smooth.

Definition. A morphism (also called a smooth map) of differentiable spaces is a continuous map which pulls back smooth functions to smooth functions. That is, $h: X \rightarrow Y$ is smooth if

1. $h$ is continuous,

2. For all open $U \subset Y$ and $f \in C^{\infty}(U), f \circ h \in C^{\infty}\left(h^{-1} U\right)$.

Remark. Condition 1 is superfluous if $Y$ happens to have the topology generated by sets of the form $f^{-1}(V)\left(V\right.$ open in $\left.\mathbf{R}, f \in C^{\infty}(Y)\right)$.

The category of differentiable spaces will be denoted $\mathscr{D}$.

Example 6. If $M$ and $N$ are smooth manifolds, then $f: M \rightarrow N$ is a morphism of differentiable spaces if and only if it is a smooth map in the usual sense.

Example 7. Let $M$ be a smooth manifold, let $\Gamma$ be a Lie groupoid, let $\left\{U_{i}\right\}(i \in \mathbf{N})$ be an open cover of $M$, and let $\left\{\gamma_{i j}: U_{i} \cap U_{j} \rightarrow \Gamma\right\}$ be a cocycle 
on $M$ with values in $\Gamma$ having smooth transition functions $\gamma_{i j}$. (This is the case, for example, if $\Gamma=\Gamma_{q}$ and $\left\{\gamma_{i j}\right\}$ is the Haefliger cocycle defined by a smooth codimension $q$ foliation on $M$ ). Let $\left\{u_{i}\right\}$ be a smooth partition of unity subordinate to $\left\{U_{i}\right\}$. The cocycle can be classified by the map

$$
\begin{gathered}
f: M \rightarrow B \Gamma, \\
f(x)=\left\{u_{i}(x), \gamma_{i j}(x)\right\}
\end{gathered}
$$

(see [5]). Since $f$ pulls back $t_{i}$ to $u_{i}$ and $g_{i j}$ to $\gamma_{i j}$, it is a morphism of differentiable spaces.

Definition. Let $X$ be a differentiable space, and $Y$ a topological subspace of $X$. One makes $Y$ a differentiable subspace of $X$ by defining

if $V$ is open in $Y$ and $f: V \rightarrow \mathbf{R}$, then $f \in C^{\infty}(V)$ if and only if for each $y \in V$ there exist a neighborhood $U$ of $y$ in $X$ and an element $g \in C^{\infty}(U)$ such that $g|U \cap V=f| U \cap V$.

We can give a global description of $C^{\infty}(Y)$ if certain smooth partitions of unity exist.

Definition. Let $X$ be a differentiable space, and $\left\{U_{\alpha}\right\}$ an open cover of $X$. Then a smooth partition of unity subordinate to $\left\{U_{\alpha}\right\}$ is (as usual) a collection $\left\{f_{\alpha} \in C^{\infty}(X)\right\}$ satisfying

(i) $f_{\alpha} \geqslant 0$ and $\Sigma f_{\alpha}=1$,

(ii) $\operatorname{supp}\left(f_{\alpha}\right)=($ def. $) \mathrm{Cl}\left(f^{-1}(0, \infty)\right) \subset U_{\alpha}$,

(iii) the collection $\left\{\operatorname{supp} f_{\alpha}\right\}$ is locally finite.

Theorem 1.1. (i) Let $X$ be a differentiable space, and let $Y \subset X$. Suppose that every open neighborhood $W$ of $Y$ admits smooth partitions of unity subordinate to any open cover (of $W$ ). Then $f \in C^{\infty}(Y)$ if and only if $f$ extends to a smooth function on some open neighborhood $U$ of $Y$.

(ii) If $X$ admits smooth partitions of unity, and $Y \subset X$ is closed, then $Y$ admits smooth partitions of unity, and every $f \in C^{\infty}(Y)$ extends (non-uniquely, in general) to some $f_{1} \in C^{\infty}(X)$.

Proof. (i) For each $y \in Y$ choose a neighborhood $U_{y}$ of $y$ in $X$ and an $f_{y} \in C^{\infty}\left(U_{y}\right)$ such that $f_{y}\left|U_{y} \cap Y=f\right| U_{y} \cap Y$. Let $U=U_{y} U_{y}$, and let $\left\{u_{y}\right\}$ be a smooth partition of unity on $U$ subordinate to $\left\{U_{y}\right\}$. Then $\Sigma_{y} u_{y} f_{y}$ is a smooth extension of $f$ to $U$.

(ii) For the second assertion, proceed as in the proof of (i), but add the open set $X-Y$ to the collection $\left\{U_{y}\right\}$ to get a cover of $X$. Choosing a partition of unity, we extend $f$ to $\Sigma u_{y} f_{y}$ as before. A similar construction shows that $Y$ admits smooth partitions of unity.

Example 8. If $K \subset \mathbf{R}^{n}$, then $f: K \rightarrow \mathbf{R}$ is smooth if and only if $f$ extends to some neighborhood of $K$ in $\mathbf{R}^{n}$. It is easy to see that if $K$ has an affine or 
convex structure (e.g., $K=\Delta^{k}$, the Euclidean $k$-simplex), then $C^{\infty}(K)$ is independent of $n$ and of the affine embedding of $K$ in $\mathbf{R}^{n}$.

Example 9. If $X$ is any differentiable space and $U$ an open subspace, then $\mathbf{C}^{\infty} U=\mathbf{C}^{\infty} X \mid U$.

\section{The de Rham complex of a differentiable space}

Rather than defining a notion of tangent vector for differentiable spaces (see [25], [29]), we shall define differential forms as abstract symbols $\Sigma f_{\alpha_{0}} d f_{\alpha_{1}}$ $\wedge \cdots \wedge d f_{\alpha_{k}}\left(f_{\alpha_{i}} \in C^{\infty}(U), U \subset X\right)$, and compare forms by pulling them back to open subsets of Euclidean space.

Definition. Let $X$ be a differentiable space. Then a plot of $X$ is a smooth morphism $\phi: E \rightarrow X$, where $E$ is an open subspace of $\mathbf{R}^{n}$ for some (finite) $n$. (The terminology is adapted from Chen's [8]).

Note. For convenience, we will sometimes drop the requirement that $E$ be open, and allow plots of the form $\phi: I \rightarrow X, \phi: E \times I \rightarrow X$, etc. This will not change any results.

Definition. Let $U$ be a differentiable space, and let $f_{i j} \in C^{\infty}(U), i=$ $1, \cdots, p ; j=0, \cdots, q$. Let $\eta$ denote the symbol $\Sigma_{i=1}^{p} f_{i 0} d f_{i 1} \wedge \cdots \wedge d f_{i q}$, and let $\phi: E \rightarrow U$ be a plot. Then $\phi^{*} \eta$ will denote the differential form

$$
\sum_{i=1}^{p}\left(f_{i 0} \circ \phi\right) d\left(f_{i 1} \circ \phi\right) \wedge \cdots \wedge d\left(f_{i q} \circ \phi\right) \in A^{q}(E) .
$$

Let $B^{q}(U)$ be the real vector space of symbols of this form ( $p$ is arbitrary) modulo the equivalence relation:

$$
\eta_{1} \sim \eta_{2} \text { if and only if } \phi^{*} H_{1}=\phi^{*} \eta_{2} \text { for all plots } \phi: E \rightarrow U
$$

If $X$ is a differentiable space, then the rule $U \rightarrow B^{q}(U)$ ( $U$ open in $X$ ) defines a presheaf of real vector spaces on $X$. Let $\mathbf{A}^{q} X$ be the sheaf generated by this presheaf, and let $A^{q}(U)=\Gamma\left(\mathbf{A}^{q} X \mid U\right)=\Gamma \mathbf{A}^{q} U(\Gamma=$ sections $)$.

Remark. $B^{q}(X)$ contains finite sums of symbols $f_{0} d f_{1} \wedge \cdots \wedge d f_{q}$, but $A^{q}(X)$ also contains locally finite sums of such symbols.

Lemma 2.1. The canonical homomorphism $s: B^{q}(U) \rightarrow A^{q}(U)$ is injective.

Proof. Let $\eta \in$ ker $s$. By sheaf theory we can find an open cover $\left\{U_{\alpha}\right\}$ of $U$ such that each restriction $\eta_{\alpha} \in B^{q}\left(U_{\alpha}\right)$ of $\eta$ equals 0 . Let $\phi: E \rightarrow U$ be a plot. It suffices to show that $\phi^{*} \eta=0$. Let $L_{\alpha}=\phi^{-1}\left(U_{\alpha}\right)$. Then $\phi \mid L_{\alpha}$ is a plot of $U_{\alpha}$, and $\phi^{*} \eta \mid L_{\alpha}=\left(\phi \mid L_{\alpha}\right)^{*} \eta=\left(\phi \mid L_{\alpha}\right)^{*} \eta_{\alpha}=0$. It follows that $\phi^{*} \eta=0$.

Corollary 2.2. If $\phi: E \rightarrow X$ is a plot and $\eta \in A^{q}(X)$, then there is a well-defined form $\phi^{*} \eta \in A^{q}(E)$. Also, $\eta_{1}=\eta_{2}$ if and only if $\phi^{*} \eta_{1}=\phi^{*} \eta_{2}$ for all plots $\phi$. 
Lemma 2.3. (i) $B^{0}(U)=A^{0}(U)=C^{\infty}(U)$.

(ii) $B^{q}(U)$ and $A^{q}(U)$ are modules over $C^{\infty}(U)$ via

(iii) $f \cdot\left(f_{0} d f_{1} \wedge \cdots \wedge d f_{q}\right)=\left(f f_{0}\right) d f_{1} \wedge \cdots \wedge d f_{q}$.

(iv) $\mathbf{A}^{q} X$ is a module over the sheaf $\mathbf{C}^{\infty} X$.

(v) The exterior derivatives $d: B^{q} \rightarrow B^{q+1}$ and $d: A^{q} \rightarrow A^{q+1}$ are well-defined by

$$
d\left(f_{0} d f_{1} \wedge \cdots \wedge d f_{q}\right)=1 d f_{0} \wedge \cdots \wedge d f_{q}
$$

and satisfy $d^{2}=0$.

(vi) The wedge products $B^{p} \wedge B^{q} \rightarrow B^{p+q}$ and $A^{p} \wedge A^{q} \rightarrow A^{p+q}$ are well-defined by

$$
\left(f_{0} d f_{1} \wedge \cdots \wedge d f_{p}\right) \wedge\left(g_{0} d g_{1} \wedge \cdots \wedge d g_{p}\right)=\left(f_{0} g_{0}\right) d f_{1} \wedge \cdots \wedge d g_{q},
$$

and are graded-commutative.

Proof. To see that each operation is well-defined, pull back the forms in question to $A^{*}(E)$ via plots $\phi: E \rightarrow X$ and use Corollary 2.2.

Definition. The commutative differential graded R-algebra $A^{*}(X)=$ $\bigoplus_{q} A^{q}(X)$ is called the de Rham complex of the differentiable space $X$. The de Rham cohomology of $X$ is defined by

$$
H_{D R}^{*}(X)=H_{d} A^{*}(X) \text {. }
$$

Example 10. If $X$ is a smooth manifold, then $A^{*}(X)$ and $H_{D R}^{*}(X)$ turn out to have their usual meaning.

Morphisms. Let $h: X \rightarrow Y$ be a morphism of differentiable spaces. Then the rule

$$
h^{*}\left(f_{0} d f_{1} \wedge \cdots \wedge d f_{q}\right)=\left(f_{0} \circ h\right) d\left(f_{1} \circ h\right) \wedge \cdots \wedge d\left(f_{q} \circ h\right)
$$

induces algebra homomorphisms

$$
\begin{aligned}
h^{*}: A^{*}(Y) & \rightarrow A^{*}(X), \\
h^{*}: B^{*}(Y) & \rightarrow B^{*}(X), \\
h^{*}: H_{D R}^{*}(Y) & \rightarrow H_{D R}^{*}(X) .
\end{aligned}
$$

Lemma 2.4 (The chain rule). Let $X$ be a differentiable space, let $f_{1}, \cdots, f_{n}$ $\in C^{\infty}(X)$, and let $g \in C^{\infty}(V), V$ open in $\mathbf{R}^{n}$, with $V \supset$ image $\left(f_{1}, \cdots, f_{n}\right)$ : $X \rightarrow \mathbf{R}^{n}$. Then

$d\left(g \circ\left(f_{1}, \cdots, f_{n}\right)\right)=\sum_{i=1}^{n}\left[\left(D_{i} g\right) \circ\left(f_{1}, \cdots, f_{n}\right)\right] \cdot d f_{i} \in A^{1}(X),\left(D_{i}-\frac{\partial}{\partial x_{i}}\right)$.

Proof. Pull back both sides of the asserted identity via plots $\phi: E \rightarrow X$. Since the Chain Rule is valid on manifolds, the pullbacks of both sides are equal. Corollary 2.2 now completes the proof. 


\section{Smooth homotopy invariance}

Products. Let $X$ and $Y$ be differentiable spaces. Then $X \times Y$ is the differentiable space defined by giving $X \times Y$ the usual Cartesian product topology and defining

for $U$ open in $X \times Y, C^{\infty}(U)=\{f: U \rightarrow \mathbf{R} \mid f$ is locally of the form $g \circ\left(f_{1}, \cdots, f_{n}\right)$, where $g \in C^{\infty}\left(\mathbf{R}^{n}\right)$ ( $n$ arbitrary) and each $f_{i}$ belongs to $C^{\infty}(V)$ (for some open $V \subset X$ ) or to $\left.C^{\infty}(W)(W \subset Y)\right\}$.

Example. Let $I$ be the unit interval, regarded as a differentiable subspace of $\mathbf{R}$ with coordinate $t$. Then $f \in C^{\infty}(X \times I)$ if and only if $f$ is locally of the form $g \circ\left(t, f_{1}, \cdots, f_{n}\right)$, where $g \in C^{\infty}\left(\mathbf{R}^{n+1}\right)$ and $f_{i} \in C^{\infty}(U)(U \subset X)$.

Definition. A smooth homotopy is a morphism of differentiable spaces $F=\left\{f_{t}\right\}: X \times I \rightarrow Y$; one says that $f_{0}$ and $f_{1}$ are smoothly homotopic.

By mimicking the proof of smooth homotopy invariance of de Rham cohomology on manifolds [38], we now prove that $H_{D R}^{*}$ is smooth homotopy invariant on the larger category $\mathscr{D}$ of differentiable spaces. We start with two technical lemmas which in the manifold case are proved by choosing coordinates.

Lemma 3.1. Let $\eta \in A^{q}(X \times I)$ ( $X$ a differentiable space). Then every $x \in X$ has a neighborhood $U$ in $X$ such that $\eta \mid U \times I$ can be written as a finite sum of terms of the form $a(x, t) d t \wedge d f_{1} \wedge \cdots \wedge d f_{q-1}$ and $b(x, t) d f_{1}$ $\wedge \cdots \wedge d f_{q}\left(a, b \in C^{\infty}(U \times I), f_{i} \in C^{\infty}(U)\right)$.

Proof. By the definition of $\mathbf{C}^{\infty}(X \times I)$ and the Chain Rule, $\eta$ is locally (on $X \times I$ ) a finite sum of terms of the desired form. Cover $\{x\} \times I$ by a finite number of product opens $U_{\alpha} \times J_{\alpha} \subset X \times I$ on each of which $\eta$ has such a representation $\eta_{\alpha}$. Let $U=\cap U_{\alpha}$, and let $\left\{j_{\alpha}\right\}$ be a smooth partition of unity subordinate to the cover $\left\{J_{\alpha}\right\}$ of $I$. Then $\Sigma_{\alpha} j_{\alpha} \eta_{\alpha}$ is the desired representation of $\eta$ on $U \times I$.

Lemma 3.2. Let $f \in C^{\infty}(X \times I)$ ( $X$ a differentiable space). Define $F$ : $X \times I \rightarrow \mathbf{R}$ by

$$
F(x, u)=\int_{0}^{u} f(x, t) d t .
$$

Then $F \in C^{\infty}(X \times I)$, and hence $\int_{0}^{1} f(x, t) d t \in C^{\infty}(X)$.

Proof. Choose $x \in X$. By an argument like that used to prove Lemma 3.1, we can find a neighborhood $U$ of $x$ in $X$ such that $f \mid U \times I=$ $g \circ\left(t, f_{1}, \cdots, f_{n}\right)$, where $g \in C^{\infty}\left(\mathbf{R}^{n+1}\right)$ and $f_{i} \in C^{\infty}(U)$. Define $G: \mathbf{R}^{n+1} \rightarrow$ $\mathbf{R}$ by

$$
G\left(u, x_{1}, \cdots, x_{n}\right)=\int_{0}^{u} g\left(t, x_{1}, \cdots, x_{n}\right) d t
$$


Then $G \in C^{\infty}\left(\mathbf{R}^{n+1}\right)$, and $F(x, u)=G\left(u, f_{1}(x), \cdots, f_{n}(x)\right)$, so that $F=$ $G \circ\left(u, f_{1}, \cdots, f_{n}\right) \in C^{\infty}(X \times I)$. q.éd.

We can now construct a homotopy operator

$$
L: A^{q}(X \times I) \rightarrow A^{q-1}(X)
$$

between $i_{0}^{*}$ and $I_{1}^{*}: A^{*}(X \times I) \rightarrow A^{*}(X)$ (where $i_{j}: X \rightarrow X \times\{j\} \subset X \times I$, $j=0,1)$ by the usual formula $[38$, p. 124]

$$
\begin{aligned}
L\left(a(x, t) d t \wedge d f_{1} \wedge \cdots \wedge d f_{q-1}\right) & =\left(\int_{0}^{1} a(x, t) d t\right) d f_{1} \wedge \cdots \wedge d f_{q-1}, \\
L\left(b(x, t) d f_{1} \wedge \cdots \wedge d f_{1}\right) & =0
\end{aligned}
$$

$\left(f_{i} \in C^{\infty}(U)\right.$ ). To see that this definition of $L$ is independent of choices, it suffices to pull back via plots of the form $\phi \times$ id: $E \times I \rightarrow X \times I$, and to observe that the operator $L^{\prime}: A^{q}(E \times I) \rightarrow A^{q-1}(E)$ defined by the same formula as $L$ is known to be well-defined [38]. The same argument shows that $d L+L d=i_{1}^{*}-i_{0}^{*}$. Hence we have proved

Theorem 3.3. The functor $H_{D R}^{*}$ (on differentiable spaces) is invariant under smooth homotopies.

\section{Comparison with other theories}

The idea of studying differential forms on non-manifolds by looking at their pullbacks to manifolds goes back to the calculus of variations. A number of authors have exploited this idea to define various notions of differentiable spaces and de Rham complexes, with the goal of computing the real cohomology of a space. In this section we will compare some of these theories briefly with emphasis on their utility as cochain theories on various spaces. We should mention that the term "differentiable space" has also been used in a number of quite different senses (including as a synonym for smooth manifold), which we shall not discuss in this section.

Closest to our theory are those of J. W. Smith [30] and K. T. Chen [9]. Smith defined a differentiable space to be a topological space $X$ together with a collection $C^{\infty}(X)$ of continuous $\mathrm{R}$-valued functions on $X$ satisfying the closure condition:

If $f: X \rightarrow \mathbf{R}$ is continuous and if $f \circ \phi \in C^{\infty}(U)$ for all plots $\phi: U \rightarrow X$ ( $U$ open in some $\mathbf{R}^{n}$, plot defined as in $\left.\S 2\right)$, then $f \in C^{\infty}(X)$.

This closure condition is stronger than ours. For example, if we regard $\mathbf{Q}$ (the rationals) as a differentiable subspace of $\mathbf{R}$, then Smith's $C^{\infty}(\mathbf{Q})$ consists of all continuous functions on $\mathbf{Q}$, while our $C^{\infty}(\mathbf{Q})$ contains only those func- 
tions which locally are the restrictions of smooth functions on $\mathbf{R}$. Smith, like us, defines differential forms as symbols which are locally of the form $\Sigma f_{0} d f_{1} \wedge, \ldots, \wedge d f_{q}$, but he interprets them as singular cochains by integrating them over cubical simplices. Our two approaches to forms are equivalent, however, since a differential form on $\mathbf{R}^{n}$ is nonzero if and only if its integral over some smooth simplex of $\mathbf{R}^{n}$ is nonzero. Smith distinguishes two notions of homotopy, roughly corresponding to using the two different closure conditions in defining $C^{\infty}(X \times I)$. His $H_{D R}^{*}$ is proved invariant only with respect to what he calls proper homotopies, corresponding to the weaker closer condition. It is partially for this reason that we prefer to use the weaker closure condition in the definition of differentiable space.

K. T. Chen focuses his attention on the plots of $X$ rather than on $C^{\infty}(X)$. He defines [9] a differentiable space to be a topological space $X$ (the topology is not essential for the construction) together with a collection of (continuous) maps $\phi: U \rightarrow X\left(U\right.$ a convex region $=($ def. $)$ closed convex set $\subset \mathbf{R}^{n}$, some $\left.n\right)$ called plots, satisfying the closure conditions:

(i) If $U^{\prime}$ is a convex region, $g: U^{\prime} \rightarrow U$ is $C^{\infty}$, and $\phi: U \rightarrow X$ is a plot, then $\phi g$ is a plot.

(ii) Every constant map $U \rightarrow X$ is a plot.

(iii) Let $\left\{g_{i}: U_{i} \rightarrow U\right\}$ be a family of maps ( $U_{i}$ and $U$ being convex regions) such that a function $f: U \rightarrow \mathbf{R}$ is $C^{\infty}$ if and only if $f g_{i}$ is $C^{\infty}$ for all $i$. If $\phi$ : $U \rightarrow X$ is a continuous map and each $\phi \circ g_{i}$ is a plot, then $\phi$ is a plot.

A $q$-form $\eta$ on $X$ is then defined to be a collection of forms $\left\{\eta_{\phi} \in\right.$ $A^{q}(U) \mid \phi: U \rightarrow X$ a plot $\}$ which are compatible with respect to pulling back via smooth maps $g: U^{\prime} \rightarrow U$. For example,

$$
C^{\infty}(\mathbf{Q})=A^{0}(\mathbf{Q})=\{\text { set maps } f: \mathbf{Q} \rightarrow \mathbf{R}\} .
$$

We have seen that $C^{\infty}(\mathbf{Q})$ is different in all three theories discussed so far. In fact, one can show that $H_{D R}^{0}(Q)=C^{\infty}(Q)$ in all three theories, so that the theories are inequivalent at the cohomology as well as the cochain level. Only in Chen's theory does $H_{D R}^{0}(\mathbf{Q})$ agree with $H_{\text {sing }}^{*}(\mathbf{Q} ; \mathbf{R})$, essentially because his theory ignores the topology of $\mathbf{Q}$.

In all three theories, a differential form is defined by its pullbacks to finite dimensional Euclidean spaces. Since such pullbacks are compatible, we see that Chen's de Rham complex contains Smith's and ours provided that one chooses for the plots in Chen's definition those continuous maps $\phi: U \rightarrow X$ ( $U$ a convex region) which pull back each $C^{\infty}$ function on $X$ (as chosen in Smith's theory) to a $C^{\infty}$ function on $U$. In specific applications one often wants to use a de Rham complex smaller than the full Chen complex so that one can give explicit formulas for differential forms. Using Smith's or our 
construction is one way to do this, or one can use special constructions for particular spaces, such as Chen's iterated integrals on path spaces [8], [9].

The choice of a differentiable space theory depends on the use to be made of it. Chen's theory is well-suited to studying path spaces $P(Y)$, since a plot $\phi$ : $K \rightarrow P(Y)$ is just a smooth map $K \times I \rightarrow Y$. Smith used his theory to study quotients of manifolds. On a space that comes equipped with a distinguished set of functions, for example a simplicial complex with its barycentric coordinates, or $B G$ with the functions $t_{i}$ and $g_{i j}$ (see $\S 1$ ), it is natural to apply Smith's or our theory. In practice, Smith's closure condition can be difficult to verify on an explicit collection of functions, and therefore we prefer our own definition for studying simplicial complexes, classifying spaces, and geometric realizations.

The definition which we have given of differentiable space appeared in the work of R. Sikorski [29], but he did not define differential forms in the way we did. Rather, he defined tangent vector fields on $X$ as derivations of the ring $C^{\infty}(X)$, and did not define a de Rham theory. (A de Rham theory based on the same definition of vector field was worked out by R. Palais [25].) Thus the individual elements of our definition have appeared elsewhere. Nonetheless, we believe that their combination is new, and that the idea of treating simplicial complexes, Milnor's $B G$, and geometric realizations of semi-simplicial spaces as differentiable spaces, using a category of differentiable spaces so general that it includes morphisms like $f: M \rightarrow B G$ ( $M$ an ordinary manifold, $f$ the classifying map of a $G$-bundle on $M$ ), is new.

A different way of putting differential forms on simplicial complexes is to take compatible collections of forms on the simplices. This was done by Whitney [40, p. 226] and Thom [34] (in the special case of a triangulated manifold, the zero-forms obtained are the piecewise smooth (semi-smooth) functions [19, p. 5]), and the idea has been refined by Sullivan [33] and others to compute not only the real cohomology but also the rational homotopy type of a simplicial complex. In a similar vein, J. Dupont [14] and C. Watkiss [37] defined differential forms on classifying spaces $B G$ and geometric realizations $\|X\|$ of semi-simplical manifolds as collections of differential forms on $G^{n} \times \Delta^{n}$ (resp. $X_{n} \times \Delta^{n}$ ) compatible under the face maps. The major difference between these theories and ours is that compatible collection theories require working in simplicial or semi-simplicial categories which do not include morphisms like $f: M \rightarrow B G$, while our theory mixes simplicial and non-simplicial constructions easily. For example, in our theory the homomorphism $f^{*}: A^{*} B G \rightarrow A^{*} M$ is defined at the cochain level of differential forms, while in the compatible collection approach $f^{*}$ is defined only at the cohomology level or as a map of Čech-de Rham double complexes. 
A more detailed comparison of our de Rham complex with those of Whitney, Dupont, and Watkiss will be given in $\$ \S 7$ and 9.

So far we have discussed only those de Rham theories based on objects which look like differential forms on the actual spaces in question. For example, in Dupont's, Watkiss', and our theories, a form on $B G$ involves the barycentric coordinates $t_{i}$ as well as the $G$-coordinates $g_{i j}$. If one's purpose is to compute the cohomology of a space, a smaller complex may suffice. For example, Bott, Shulman, and Stasheff [3] showed that the cohomology of the double complex $\oplus_{p, q} A^{q}\left(X_{p}\right)$ equals $H_{\text {sing }}^{*}(\|X\| ; \mathbf{R})$ if a de Rham isomorphism holds on each $X_{p}$. To compute $H^{*}(B G)$ in the case when $G$ is compact it suffices to study the Weil algebra [6] or the semi-simplicial Weil algebra of Kamber and Tondeur [17].

While these theories are probably easier to use than differentiable spaces to compute the cohomology of specific spaces like $B G$, differentiable spaces are useful for studying how this cohomology maps into the cohomology of manifolds at the cochain level, especially when a $G$-bundle on $M$ is specified by transition functions. Furthermore, the differentiable space approach, being very general, is applicable to other spaces as well.

There have been a number of other generalizations of differential forms to non-manifolds. For example, C. D. Marshall [20] defined a de Rham theory for subcartesian spaces, which are Hausdorff spaces locally homeomorphic to (not necessarily open) subspaces of $\mathbf{R}^{n}$ ( $n$ varying). In a different direction, there is a de Rham theory for infinite dimensional manifolds. These theories do not apply to Milnor classifying spaces $B G$ or to non-locally finite simplicial complexes, however, since these spaces are not locally homeomorphic to any set in $\mathbf{R}^{n}$ or to any open set in $\mathbf{R}^{\infty}$.

\section{Conditions guaranteeing a de Rham isomorphism}

Because the concept of differentiable space is so general, it is clear that a de Rham isomorphism $H_{D R}^{*}(X)=H_{\text {singular }}^{*}(X ; \mathbf{R})$ will hold only if we place some restrictions on $X$ and $\mathrm{C}^{\infty} X$. In this section we present two such sets of restrictions. The first criterion is a local property (smooth local contractibility) together with requirements of paracompactness and existence of smooth partitions of unity, and is proved using sheaf theory; a virtually identical theorem was proved by Smith for his theory [30]. The second criterion is "semi-local": if a de Rham isomorphism holds on all finite intersections of an open cover of $X$ which admits a smooth partition of unity, then it holds on $X$. This is proved by adapting A. Weil's Čech-de Rham double complex [38]. In the next section we shall prove the existence of smooth partitions of unity on 
simplicial complexes. This will allow us to apply the second criterion to simplicial complexes, classifying spaces, and geometric realizations in $\$ \S 7$ and 8.

To compare de Rham and singular cohomology, we map both of them to the smooth singular cohomology $H_{s m}$, which is defined as follows.

Definition. A smooth singular $q$-simplex of a differentiable space $X$ is a morphism $\sigma: \Delta^{q} \rightarrow X$ of differentiable spaces. The real-valued cochains on these simplices form a vector space denoted $S_{s m}^{q}(X)$; we set $H_{s m}^{q}(X)=$ $H^{q} S_{s m}^{*}(X)$.

Remark. The cochains of $H_{s m}^{*}$ are not sheafified.

A map from $A^{*}(X)$ to $S_{s m}^{*}(X)$ is defined, as usual, by integration of (pulled-back) forms over smooth simplices. This induces a map $H_{D R}^{*}(X) \rightarrow$ $H_{s m}^{*}(X)$.

Lemma 5.1. $H_{s m}^{*}$ is invariant under smooth homotopies of differentiable spaces.

Proof. The proof of homotopy invariance of ordinary singular cohomology [14, p. 45] can be used without change once one observes that the prism operator $P: S_{q}(X) \rightarrow S_{q+1}(X \times I)$ maps each smooth simplex to a sum of smooth simplices.

Definition. A differentiable space $X$ is locally smoothly contractible if for each open $U \subset X$ and each point $x \in U$ there are a neighborhood $V$ of $x$ in $U$ and a smooth homotopy $F=\left\{f_{t}\right\}: V \times I \rightarrow U$ satisfying

$$
\begin{gathered}
f_{0}=\mathrm{id}: V \subset U, \\
\left.f_{1}: V \rightarrow\{y\} \subset U \text { (for some point } y \in U\right) .
\end{gathered}
$$

Theorem 5.2. Let $X$ be a differentiable space which is paracompact and locally smoothly contractible, and which admits smooth partitions of unity subordinate to any open cover. Then the natural homomorphisms

$$
H_{D R}^{*}(X) \rightarrow H_{s m}^{*}(X) \leftarrow H_{\text {sing }}^{*}(X ; \mathbf{R})
$$

are isomorphisms.

Proof. Let $\mathbf{S}^{*}$ (resp. $\mathbf{S}_{s m}^{*}$ ) be the sheaf on $X$ generated by $U \rightarrow S_{\text {sing }}^{*}(U ; \mathbf{R})$ (resp. $U \rightarrow S_{s m}^{*}(U)$ ). Let $\mathbf{A}^{*}$ denote the sheaf $\mathbf{A}^{*} X$ of differential forms (see $\S 2)$. Now since $X$ is paracompact, the sheafified and unsheafified singular cohomology theories $H \Gamma S^{*}$ and $H S_{\text {sing }}^{*}=H^{*}(X)$ are isomorphic [4, p. 19]; similarly, $H \Gamma S_{s m}^{*}=H_{s m}^{*}(X)$. By definition, $H_{D R}^{*}(X)=H \Gamma A^{*}$.

Now since $X$ admits smooth partitions of unity, the sheaf $C^{\infty} X$ is fine. Since $\mathbf{A}^{*}, \mathbf{S}_{s m}^{*}$, and $\mathbf{S}^{*}$ are modules over $\mathbf{C}^{\infty} X$, they, too, are fine $[4, \mathrm{p} .50]$. Now the constant sheaf $\mathbf{R}$ injects into $\mathbf{A}^{*}, \mathbf{S}_{s m}^{*}$, and $\mathbf{S}^{*}$. We claim that all three complexes are resolutions of $\mathbf{R}$. Indeed, since $X$ is locally smoothly 
contractible and all three cohomology theories are smooth homotopy invariant, it follows that the stalk of the homology (derived) sheaf $[4$, p. 25] of all three cochain complexes of sheaves reduces to $H^{*}$ (point) $=\mathbf{R}$ (in dimension 0) at any point $x \in X$. But the cohomology of global sections of a resolution of a sheaf $F$ on a paracompact space $X$ by fine sheaves equals the sheaf cohomology $H^{*}(X ; \mathrm{F})[4, \mathrm{pp} .34,49-50]$. It follows that all these cohomologies agree, i.e.,

$$
H_{D R}^{*}(X)=H_{s m}^{*}(X)=H \Gamma \mathbf{S}_{s m}^{*}=H_{\text {sing }}^{*}(X)=H \Gamma \mathbf{S}^{*}=H_{\text {sheaf }}^{*}(X ; \mathbf{R}) \text {. q.e.d. }
$$

Our next criterion for a singular-de Rham isomorphism is based upon computing the cohomology (de Rham, singular, or smooth singular) of a space $X$ from the cohomology of each finite intersection of some open cover of $X$. In the de Rham case this entails looking at the Čech-de Rham double complex of A. Weil [38]. In the singular and smooth singular cases we use a Čech-singular double complex and an extended Mayer-Vietoris theorem.

Definition. Let $X$ be a topological space, and $U=\left\{U_{\alpha}\right\}_{\alpha \in J}$ an open cover of $X$, indexed by a totally ordered set $J$. Then $\mathcal{U}_{p}(p \in \mathbf{N})$ will denote the space $\amalg_{\sigma} U_{\sigma}$, where $\sigma=\left(\alpha_{0}, \alpha_{1}, \cdots, \alpha_{p}\right)$ runs over all strictly ordered $(\mathrm{p}+1)$-tuples of $J$, and $U_{\sigma}=U_{\alpha_{0} \cdots \alpha_{p}}=U_{\alpha_{0}} \cap \cdots U_{\alpha_{p}}$. Maps

$$
\partial_{i}: \mathcal{Q}_{p} \rightarrow \mathcal{Q}_{p-1}, i=0, \cdots, p \text {, }
$$

are defined by

$$
\partial_{i} \mid U_{\sigma}=\text { inclusion: } U_{\sigma} \subset U_{\partial_{i}} \text {, }
$$

where $\partial_{i} \sigma=\left(\alpha_{0}, \cdots, \hat{\alpha}_{i}, \cdots, \alpha_{p}\right)$.

If $X$ is a differentiable space, and $\mathscr{U}$ an open cover of $X$, then the Čech-de Rham complex of $X$, denoted $A^{*} \mathscr{Q}_{*}$, is the double complex $\bigoplus_{p, q-0} A^{q} \mathcal{Q}_{p}$ together with the Cech coboundary map

$$
\delta=\sum_{i=0}^{p+1}(-1)^{i} A^{q}\left(\partial_{i}\right): A^{q} \mho_{p} \rightarrow A^{q} \mho_{p+1}
$$

and the exterior differential $d: A^{q} \mho_{p} \rightarrow A^{q+1} \bigcup_{p}$. As usual, we make $A^{*} \mathscr{U}_{*}$ a cochain complex by defining a total differential $D=d+(-1)^{q} \delta$ and a total grading $n=p+q$.

The Čech-singular complex $S^{*} \mathscr{U}_{*}$ and $\check{C}$ ech-smooth singular complex $S_{s m}^{*} \mathcal{U}_{*}$ are defined analogously.

Remark. The relation of this definition of Čech-de Rham complex to Weil's is that a form $\eta \in A^{q} \mho_{p}$ can be identified with the alternating Čech $p$-cochain, with values in $A^{q}$, defined by $\eta_{\alpha_{0}} \cdots \alpha_{p}+ \pm \eta \mid U_{\alpha_{0}} \cdots \alpha_{p}$ where the sign is the signature of the permutation needed to put $\alpha_{0}, \cdots, \alpha_{p}$ into order. 
Theorem 5.3 (Extended Mayer-Vietoris Theorem).

(i) For any topological space $X$ and any open cover $\mathcal{Q}, H_{D}\left(S^{*} \mathcal{U}_{*}\right)=$ $H_{\text {sing }}^{*}(X ; \mathbf{R})$; the isomorphism is induced at the cochain level by the restriction map from $S^{*}(X)$ to $S^{*} \mho_{0} \subset S^{*} \mho_{*}$.

(ii) For any differentiable space $X$ and any open cover $\mathcal{Q}, H_{D}\left(S_{s m}^{*} \mathcal{U}_{*}\right)=$ $H_{s m}^{*}(X)$ induced by $S_{s m}^{*} X \rightarrow S^{*} \mathscr{Q}_{*}$.

Proof. Part (i) is more or less well-known; see [3] or [23, p. 16] for a proof. Since the proof is combinatorial in nature, it goes through for the smooth case as well; all we need to check is that the barycentric subdivision of a smooth singular simplex is a sum of smooth simplices, and this is obvious. q.e.d.

The analog of this theorem for the Čech-de Rham complex requires an additional hypothesis.

Theorem 5.4. Let $X$ be a differentiable space, and let $Q$ be an open cover of $X$ admitting a subordinate smooth partition of unity. Then

$$
H_{D}\left(A^{*} U_{*}\right)=H_{D R}^{*}(X) \text {, }
$$

induced by the restriction map $r: A^{*} X \rightarrow A^{*} \mathscr{U}_{0} \subset A^{*} \mathscr{U}_{*}$.

Proof. Let $\left\{u_{\alpha}\right\}$ be a smooth partition of unity subordinate to $\mathcal{Q}$. One first shows, as Weil did [38] (in the case where $X$ is a manifold), that the complex

$$
0 \rightarrow A^{q} X \stackrel{r}{\rightarrow} A^{q} \mathcal{U}_{0} \stackrel{\delta}{\rightarrow} A^{q} \mathcal{U}_{1} \stackrel{\delta}{\rightarrow} \cdots
$$

is exact, by using N. Hamilton's homotopy operator

$$
\begin{gathered}
N: A^{q} \bigcup_{p+1} \rightarrow A^{q} \mho_{p}, p=-1,0,1, \cdots, \\
(N \omega)_{\alpha_{0}} \cdots \alpha_{p}=\sum_{\alpha \in J} u_{\alpha} \cdot \omega_{\alpha \alpha_{0}} \cdots \alpha_{p},
\end{gathered}
$$

(here $\mathscr{U}_{-1}=X$ ) which makes sense in our context since $A^{*}(X)$ is a module over $C^{\infty}(X)$ and is closed under locally finite sums. The rest of the proof is exactly the same as Weil's.

Our second main theorem is now easy to prove.

Theorem 5.5. Let $X$ be a differentiable space, and let $\mathcal{Q}$ be an open cover of $X$ admitting a subordinate smooth partition of unity. Suppose that for every finite intersection $U_{\sigma}$ of opens in $\mathscr{Q}$, the maps

$$
H_{D R}^{*}\left(U_{\sigma}\right) \rightarrow H_{s m}^{*}\left(U_{\sigma}\right) \leftarrow H_{\text {sing }}^{*}\left(U_{\sigma} ; \mathbf{R}\right)
$$

are isomorphisms. Then the maps

$$
H_{D R}^{*}(X) \rightarrow H_{s m}^{*}(X) \leftarrow H_{\text {sing }}^{*}(X ; \mathbf{R})
$$

are isomorphisms. 
Proof. Consider the maps of double complexes

$$
A^{*} \mathscr{U}_{*} \rightarrow S_{s m}^{*} \mathscr{U}_{*} \leftarrow S^{*} \mho_{*}
$$

Filtering by $p$ we get spectral sequences which map at the $E_{1}$ level by

$$
H_{d}^{q}\left(A^{*} \mho_{p}\right)=H_{D R}^{q}\left(\mho_{p}\right) \rightarrow H_{s m}^{q}\left(\mho_{p}\right) \leftarrow H_{\text {sing }}^{q}\left(\mho_{p}\right) \text {. }
$$

By hypothesis these maps are isomorphisms, so

$$
H_{D}\left(A^{*} \mathscr{Q}_{*}\right) \underset{\rightarrow}{\approx} H_{D}\left(S_{s m}^{*} \mathscr{Q}_{*}\right) \approx H_{D}^{*}\left(S^{*} \mathscr{Q}_{*}\right)
$$

by the usual spectral sequence arguments. Theorems 5.3 and 5.4 now yield the desired conclusion.

\section{Smooth partitions of unity}

In order to apply Theorems 5.2 and 5.5 we need to known when an open cover of a differentiable space admits a subordinate smooth partition of unity. In this section we find some convenient sufficient conditions for smooth partitions of unity to exist.

The existence of partitions of unity on Banach spaces has been studied extensively, and some of the results obtained for Banach spaces are general enough to apply to differentiable spaces. In a recent paper [36], H. Torunczyk gave what amount to necessary and sufficient conditions for a differentiable space whose topology is metrizable to admit smooth partitions of unity. First we need the following definitions.

Definition. Let $X$ be a differentiable space. Then $\mathcal{Q}_{X}$ will denote the collection of open subsets of $X$ of the form $f^{-1}(a, b)$ where $f \in C^{\infty}(X), a$, $b \in \mathbf{R}$.

Definition. A family of subsets is $\sigma$-locally finite if it is the union of a countable number of locally finite subfamilies.

Definition. Given a set $A, c_{0}(A)$ is the linear space of all $x=\left(x_{a}\right) \in \mathbf{R}^{A}$ with $\left\{a \in A:\left|x_{a}\right|>1 / n\right\}$ finite for any integer $n \geqslant 1 ; c_{0}(A)$ is regarded as a Banach space under the norm $\left\|\left(x_{a}\right)\right\|=\sup \left\{\left|x_{a}\right|: a \in A\right\}$. We make $c_{0}(A)$ a differentiable space by defining $f \in C^{\infty}(U)$ ( $U$ open in $c_{0}(A)$ ) if and only if $f$ is locally a smooth function of finitely many coordinate functions $x_{a_{1}}, \cdots, x_{a_{n}}$.

Theorem 6.1 (H. Torunczyk [36]). The following conditions are equivalent for a differentiable space $X$ whose underlying topological space is metrizable.

(a) $X$ admits smooth partitions of unity (subordinate to any open cover).

(b) $\mathcal{U}_{X}$ contains a $\sigma$-locally finite base of the topology of $X$.

(c) There are $a$ set $A$ and $a$ homeomorphic embedding $u: X \rightarrow c_{0}(A)$ with $x_{a} \circ u \in C^{\infty}(X)$ for any $a \in A$. 
Remarks. 1. Condition (b), together with the stipulation that $C^{\infty}(X)$ separate the points of $X$ (so that $X$ is $T_{3}$ ), implies that $X$ is metrizable [13, p. 194]. Condition (c) also implies that $X$ is metrizable since $c_{0}(A)$ is a metric space.

2. The map $u$ in part (c) is a morphism of differentiable spaces.

An important special case of this theorem is the following.

Theorem 6.2. Let $X$ be a differentiable space. Suppose that $C^{\infty}(X)$ contains a countable collection $f_{0}, f_{1}, f_{2} \cdots$ which separates the points of $X$ and generates the topology of $X$ (in the sense that the sets $f_{i}^{-1}(a, b)$ are a sub-basis of the topology of $X$ ). Then $X$ admits smooth partitions of unity subordinate to any open cover.

Proof. The collection $\left\{f_{i}^{-1}(a, b): i \in \mathbf{N}, a, b\right.$ rational $\}$ is a countable sub-basis for $X$. The collection $\mathscr{Q}_{X}$ of opens is closed under finite intersections [36], hence includes the countable subcollection consisting of all finite intersections of the $f_{i}^{-1}(a, b)$. Thus $\mathcal{Q}_{X}$ contains a countable (hence $\sigma$-locally finite) basis of $X$. Now apply Theorem 6.1 and Remark 1 .

\section{Applications of Theorems 6.1 and 6.2}

Theorem 6.3. Let $X$ be a simplicial complex in the strong (metric) topology, regarded as a differentiable space (see $\$ 1$, Example 5). Then $X$ admits smooth partitions of unity subordinate to any open cover.

Proof. Let $A$ be the set of vertices of $X$, and let $\left\{t_{a}\right\}(a \in A)$ be the barycentric coordinates. Define $u: X \rightarrow c_{0}(A)$ by $u(x)=\left(t_{a}(x)\right)$. One verifies easily (see Remark) that $u$ is a homeomorphic embedding, and it is trivial that $x_{a} \circ u=t_{a} \in C^{\infty}(X)$. Now apply Theorem 6.1.

Remark. The strong topology on a simplicial complex $X$ with vertex set $A$ is defined in any of the following three equivalent ways:

(1) $\left\{t_{a}^{-1}(c, d)\right\}(a \in A, c, d \in \mathbf{R})$ is a sub-basis for the topology of $X$.

(2) $X$ is a metric space with metric $p_{1}(x, y)=\left(\Sigma_{a}\left(t_{a}(x)-t_{a}(y)\right)^{2}\right)^{\frac{1}{2}}$.

(3) $X$ is a metric space with metric $p_{2}(x, y)=\sup _{a}\left|t_{a}(x)-t_{a}(y)\right|$.

The equivalence $1 \Leftrightarrow 2$ is well-known and easily proved; the equivalence $2 \Leftrightarrow 3$ can be proved easily using the identity $\Sigma t_{a}(x)=1$.

Theorem 6.4. The infinite-dimensional Lie groupoid $J_{q}$ (see $\$ 1$, Example 4) admits smooth partitions of unity.

Proof. The coordinates $x^{i}, y^{i}, y_{\alpha}^{i}\left(\alpha=\left(\alpha_{1}, \cdots, \alpha_{n}\right)\right)$ (ibid.) are a countable collection of smooth functions on $J_{q}$ which separate the points of $J_{q}$ and generate its topology. Now apply Theorem 6.2.

Theorem 6.5. Let $X$ be a differentiable space admitting smooth partitions of unity and having a metrizable topology. Then any topological subspace $Y \subset X$ with its induced structure of differentiable subspace (see §1) admits smooth partitions of unity. 
Proof. By Theorem 6.1, $X$ admits a smooth embedding $u: X \rightarrow c_{0}(A)$ for some $A$. Then $u \mid Y$ is a smooth embedding of $Y$ into $c_{0}(A)$, so that $Y$ admits smooth partitions of unity, again by Theorem 6.1.

Remark. For $Y$ closed this result is obvious (see Theorem 1.1), but Theorem 6.5 applies to any subspace $Y$.

Although we have already shown that every simplicial complex (in the strong topology) admits smooth partitions of unity, it is of interest to exhibit one explicitly in a special case. In particular, consider the infinite Euclidean simplex $\Delta^{\infty}$ in the strong topology with vertices $0,1,2, \cdots$ and barycentric coordinates $t_{0}, t_{1}, t_{2}, \cdots$. Let $U_{i}=\left\{t_{i}>0\right\}$; then $\left\{U_{i}\right\}$ is an open cover of $\Delta^{\infty}$. Now $\left\{t_{i}\right\}$ is a point-finite (but not locally finite) partition of unity, with $\left\{t_{i}>0\right\} \subset U_{i}$.

Following [10], we can construct a locally finite (but only continuous) partition of unity $\left\{u_{i}\right\}$ with $\left\{u_{i}>0\right\} \subset U_{i}$ by setting

$$
\begin{gathered}
u_{i}^{\prime}=\max \left(0, t_{i}-\sum_{j<i} t_{j}\right), \\
u_{i}=u_{i}^{\prime} / \sum_{j=0}^{\infty} u_{j}^{\prime} \text { (the sum is locally finite). }
\end{gathered}
$$

If we replace $\max (0, s)$ by any smooth function $g(s)$ which is zero for $s<0$ and positive for $s>0$, then we get a smooth partition of unity $\left\{v_{i}\right\}$ instead of $\left\{u_{i}\right\}$, but still only with $\left\{v_{i}>0\right\} \subset U_{i}$. To construct a smooth partition of unity $\left\{w_{i}\right\}$ subordinate to $\left\{U_{i}\right\}$, we must have the stronger condition $C l\left(\left\{w_{i}>\right.\right.$ $0\}) \subset U_{i}$. To obtain this, let $g$ be as before, and set

$$
w_{i}^{\prime}=g\left(v_{i}-(1 / 2)^{i+2}\right), w_{i}=w_{i}^{\prime} / \sum_{j=0}^{\infty} w_{i}^{\prime} .
$$

This is defined since if $w_{i}^{\prime}(x)=0$ for all $i$, then $v_{i}(x)<(1 / 2)^{i+2}$, so that

$$
1=\Sigma_{i} v_{i}(x) \leqslant \sum_{i=0}^{\infty}(1 / 2)^{i+2}=\frac{1}{2},
$$

contradiction. We see that

$$
\operatorname{supp}\left(w_{i}\right)=C l\left(\left\{v_{i}>(1 / 2)^{i+2}\right\}\right) \subset\left\{v_{i}>0\right\} \subset U_{i},
$$

so that $\left\{w_{i}\right\}$ is subordinate to $\left\{U_{i}\right\}$, and $\left\{\operatorname{supp}\left(w_{i}\right)\right\}_{i}$ is locally finite. Hence $\left\{w_{i}\right\}$ is a smooth partition of unity on $\Delta^{\infty}$ subordinate to $\left\{U_{i}\right\}$.

\section{De Rham cohomology of simplicial complexes}

In this section we show that the differentiable space de Rham complex of any simplicial complex $X$, using either the weak or the strong topology on $X$, 
computes the real cohomology of $X$. We compare these cochain complexes with Whitney's complex of compatible forms.

We start by recalling some topological facts about simplicial complexes. Let $X$ be a simplicial complex, and $B$ its set of vertices. As we have remarked, there are two topologies on $X$, weak and strong; we shall designate these by $X_{w}$ and $X_{s}$ respectively. Recall that $U \subset X_{w}$ is open if and only if $U \cap S$ is (relatively) open in $S$ for every (finite) simplex $S$ of $X$. On the other hand, the strong topology on $X$ is defined as the coarsest topology (fewest open sets) making all the barycentric coordinate functions $t_{b}(b \in B)$ continuous; equivalently, it is defined by the metric $p(x, y)=\left(\Sigma_{b}\left(t_{b}(x)-t_{b}(y)\right)^{2}\right)^{\frac{1}{2}} . X$ is paracompact in either topology. It is clear that id: $X_{w} \rightarrow X_{s}$ is continuous. Dowker [12] showed that $X_{w}$ and $X_{s}$ are homotopy equivalent. We recall the explicit homotopy inverse $u: X_{s} \rightarrow X_{w}$ constructed by Milnor [22]. Let $\left\{u_{b}\right\}$ be a (continuous) partition of unity subordinate to the star open cover $\left\{U_{b}\right\}_{b \in B}$ of $X_{s}\left(U_{b}=\left\{t_{b}>0\right\}\right)$.

Define $u: X_{s} \rightarrow X_{w}$ by

$$
u(x)=\left(u_{b}(x)\right)_{b \in B} .
$$

Then $u$ is continuous and maps some open neighborhood of each $x \in X_{s}$ into some finite subcomplex of $X$; it also maps each simplex of $X$ into itself. Let $h=\left\{h_{t}\right\}(0 \leqslant t \leqslant 1)$ be the linear homotopy with $h_{0}=\mathrm{id}_{X}$ and $h_{1}=u$. Then $h: X_{w} \times I \rightarrow X_{w}$ and $h: X_{s} \times I \rightarrow X_{s}$ are continuous [22], which shows that $u$ is indeed a homotopy inverse to id.

The preceding discussion can be adapted as follows to show that id: $X_{w} \rightarrow$ $X_{s}$ is a smooth homotopy equivalence of differentiable spaces. In light of Theorem 6.3, we can choose $\left\{u_{b}\right\}$ to be a smooth partition of unity. Defining $u$ as before, we see that $t_{b} \circ u=u_{b}$, so that $u$ is a smooth morphism of differentiable spaces. Also, $t_{b} \circ h=(1-t) \cdot t_{b}+t \cdot u_{b}$, which is a smooth function on both $X_{w} \times I$ and $X_{s} \times I$. Hence $h$ is smooth with respect to either topology. Thus we have proved

Theorem 7.1. Let $X$ be a simplicial complex. Then id: $X_{w} \rightarrow X_{s}$ is a smooth homotopy equivalence of differentiable spaces.

With the aid of the results of $\$ 5$, we can now prove the de Rham theorem for simplicial complexes.

Theorem 7.2. Let $X$ be a simplicial complex, in either the weak or the strong topology. Then the maps

$$
H_{D R}^{*}(X) \rightarrow H_{s m}^{*}(X) \leftarrow H_{\text {sing }}^{*}(X)
$$

are isomorphisms.

Proof. By Theorem 7.1 and the smooth homotopy invariance of all three 
cohomology theories, it suffices to consider the case $X=X_{s}$. Now let $U_{b}=$ $\left\{t_{b}>0\right\}$ (=star (b)) as before, and for each abstract simplex $\sigma=$ $\left(b_{0}, \cdots, b_{n}\right)$ of $X$ denote $\bigcap_{b \in \sigma} U_{b}$ by $U_{\sigma}$. Then $U_{\sigma}=\operatorname{star}(\sigma)$, and there is a linear deformation retraction $h$ of $U_{\sigma}$ onto the barycenter $x_{\sigma}$ of $S_{\sigma}$ (the geometric simplex corresponding to $\sigma$ ) defined by

$$
\begin{gathered}
h: U_{\sigma} \times I \rightarrow U_{\sigma}, \\
t_{b}(h(x, t))= \begin{cases}(1-t) t_{b}(x)+t /(n+1) & \text { if } b \in \sigma, \\
(1-t) t_{b}(x) & \text { if } b \notin \sigma .\end{cases}
\end{gathered}
$$

Furthermore, the explicit formulas show that $h$ is smooth. It follows by smooth homotopy invariance that $H^{*}\left(U_{o}\right)=H^{*}(\mathrm{pt})=.\mathrm{R}$ for $H=H_{D R}, H_{s m}$, and $H_{\text {sing. }}$. If we now apply Theorem 5.5, using the open cover $\left\{U_{b}\right\}$ with subordinate smooth partition of unity $\left\{u_{b}\right\}$, the theorem follows immediately. q.e.d.

Theorem 7.2 shows that $A^{*}\left(X_{s}\right)$ and $A^{*}\left(X_{w}\right)$ are commutative cochain models for the real cohomology of any simplicial complex $X$. We now ask how these cochain complexes are related to each other and to the Whitney model $A_{c}^{*}(X)$ of compatible forms on the simplices of $X$. Recall [39, p. 226] that a cochain $\omega \in A_{c}^{*}(X)$ is defined to be a collection $\left\{\omega_{\sigma} \in A^{*}\left(S_{\sigma}\right)\right\}$, where $\sigma$ runs over the abstract simplices of $X, S_{\sigma}$ is the geometric simplex corresponding to $\sigma$, and $\left\{\omega_{\sigma}\right\}$ satisfies the compatibility condition $\omega_{\sigma} \mid S_{\tau}=\omega_{\tau}$ if $\tau \subset \sigma$. Actually, Whitney worked with "flat cochains" rather than smooth differential forms, but $H A_{c}^{*}(X)=H^{*}(X ; \mathbf{R})$ in both cases (see [40] and [7] for proofs).

There are canonical homomorphisms

$$
A^{*}\left(X_{s}\right) \stackrel{\alpha}{\rightarrow} A^{*}\left(X_{w}\right) \stackrel{\beta}{\rightarrow} A_{c}^{*}(x) ;
$$

here $\alpha=\mathrm{id}^{*}$, where id: $X_{w} \rightarrow X_{s}$, while $\beta$ is defined by

$$
\beta: \omega \rightarrow\left\{\omega_{\sigma}=\omega \mid S_{\sigma}\right\} .
$$

Although $\alpha$ and $\beta$ induce isomorphisms in cohomology (as can be seen by integrating $q$-forms over $q$-simplices to map all three theories to the simplicial cohomology of $X$ ), they are not in general isomorphisms at the cochain level. The precise relationship between the three cochain complexes is given by the following theorem.

Theorem 7.3. (i) For any simplicial complex $X$, the maps $\alpha: A^{*}\left(X_{s}\right) \rightarrow$ $A^{*}\left(X_{w}\right)$ and $\beta: A^{*}\left(X_{w}\right) \rightarrow A_{c}^{*}(X)$ are injections.

(ii) If $X$ is locally finite, then $\alpha$ and $\beta$ are isomorphisms.

(iii) If $X$ is not locally finite, then $\alpha$ and $\beta$ are both strict inclusions.

The proof of this theorem is divided into Lemmas 7.4 through 7.8. 
Lemma 7.4. The map $\alpha: A^{*}\left(X_{s}\right) \rightarrow A^{*}\left(X_{w}\right)$ is injective for any simplicial complex $X$.

Proof. Observe first that the weak and strong topologies agree on finite subcomplexes of $X$, and that every plot $\phi: E \rightarrow X_{w}$ factors locally through a finite subcomplex (since $E$ is locally compact and every compact subset of $X_{w}$ is contained in a finite subcomplex [39]).

Let $\omega \in \operatorname{ker} \alpha$, and suppose that $\omega \neq 0$. Let $\phi: E \rightarrow X_{s}$ be a plot such that $\phi^{*} \omega$ is nowhere zero. (We can find such a plot by starting with a plot $\phi$ : $L \rightarrow X_{s}$ with $\phi^{*} \omega \neq 0$ and restricting suitably.) Let $X^{(n)}$ be the $n$-skeleton of $X$ (a closed subspace of $X_{s}$ ) and let $C_{n}=\phi^{-1} X^{(n)}$. Then $\left\{C_{n}\right\}$ is a countable closed cover of $E$, so by the Baire category theorem [13, p. 250] (using the local compactness of $E$ ) some $C_{m}$ has a nonempty interior $U$. Then $\phi \mid U$ : $U \rightarrow X^{(m)} \rightarrow X$ is a plot of $X_{w}$, so that $0=(\phi \mid U)^{*} \omega=\phi^{*} \omega \mid U$. This contradiction shows that $\operatorname{ker} \alpha=0$.

Lemma 7.5. The map $\beta: A^{*}\left(X_{w}\right) \rightarrow A_{c}^{*}(X)$ is injective for any simplicial complex $X$.

Proof. Let $\omega \in \operatorname{ker} \beta$, and suppose that $\omega \neq 0$. Let $\phi: E \rightarrow X_{w}$ be a plot for which $\phi^{*} \omega$ is nowhere zero. By restricting $E$ to some open subset we may assume that $\phi$ factors through a finite subcomplex $Y \subset X$. Assume that $Y$ has been chosen to be minimal (for the fixed restricted $E$ ), and let $S$ be a top-dimensional simplex of $Y$. Then $L=\left(\right.$ def.) $\phi^{-1}$ (int $\left.S\right) \subset E$ is open and nonempty. But $\phi \mid L$ factors through $S$, which implies that $0=(\phi \mid L)^{*} \omega=$ $\phi^{*} \omega \mid L$. This contradiction shows that $\operatorname{ker} \beta=0$. q.e.d.

It follows from Lemmas 7.4 and 7.5 that we can regard $A^{*}\left(X_{s}\right)$ and $A^{*}\left(X_{w}\right)$ as subcomplexes of $A^{*}{ }_{c}(X)$.

Lemma 7.6. If $X$ is a finite simplicial complex, then $A^{*}\left(X_{s}\right)=A^{*}\left(X_{w}\right)=$ $A_{c}^{*}(X)$.

Proof. The first equality follows from the fact that the strong and weak topologies agree on finite complexes; we will denote $A^{*}\left(X_{s}\right)$ and $A^{*}\left(X_{w}\right)$ by $A^{*}(X)$. For the second equality, it suffices (by Lemma 7.5) to show that $\beta$ : $A^{*}(X) \rightarrow A_{c}^{*}(X)$ is onto. We use induction on the total number $p$ of simplices of $X$. For $p=1$ we have $X=\{0\}$ and the result is obvious. Suppose the result has been proven for $p-1$, and that $X$ has $p$ simplices. Let $S$ be a top-dimensional simplex of $X$, and assume that the vertices of $S$ are labelled 0 , $1, \cdots, n$. Now $Y=\left(\right.$ def.) $X-$ int $S$ has $p-1$ simplices, so that $A^{*}(Y)=$ $A_{c}^{*}(Y)$. Let $U=\left\{\sum_{i=0}^{n} t_{i}>0\right\} \subset X$; then $U$ is a neighborhood of $S$. A smooth retraction $r: U \rightarrow S$ is defined by $t_{i}(r(x))=t_{i}(x) / \sum_{i=0}^{n} t_{i}(x), i=0$, $1, \cdots, n$. Observe that $r$ maps $U-S$ to the boundary of $S$ since $S$ is top-dimensional.

Let $\omega \in A_{c}^{*}(X)$. Then $\omega \mid Y \in A_{c}^{*}(X)=A^{*}(Y)$. By Theorems 1.1 and 6.3, 
every $f \in C^{\infty}(Y)$ extends to some $f^{\prime} \in C^{\infty}(X)$. It follows easily (using a smooth partition of unity and the compactness of $Y$ ) that $A^{*}(X) \rightarrow A^{*}(Y)$ is onto. In particular, $\omega \mid Y$ extends to some $\omega_{1} \in A^{*}(X)$. Then $\omega_{2}=\omega-\omega_{1} \in$ $A_{c}^{*}(X)$ satisfies $\omega_{2} \mid Y=0$, and to show that $\omega \in A^{*}(X)$ it suffices to show that $\omega_{2} \in A^{*}(X)$. But $\omega_{2} \mid U=r^{*}(\omega \mid S) \in A^{*} U$, while $\omega_{2} \mid$ int $Y=0$, so that $\omega_{2} \in A^{*}(X)$ as claimed.

Lemma 7.7. If $X$ is a locally finite simplicial complex, then $A^{*}\left(X_{s}\right)=$ $A^{*}\left(X_{w}\right)=A_{c}^{*}(X)$.

Proof. The question is a local one, so we can just apply Lemma 7.6 to the closures of the star opens, which are finite complexes.

Lemma 7.8. If $X$ is a simplicial complex which is not locally finite, then

$$
A^{*}\left(X_{s}\right) \varsubsetneqq A^{*}\left(X_{w}\right) \varsubsetneqq A_{c}^{*}(X) .
$$

Proof. Let 0 be a vertex contained in infinitely many 1-simplices, including, say, (01), (02), ‥ Define $f: X \rightarrow \mathbf{R}$ by

$$
f(x)=\sum_{n=1}^{\infty} n t_{n}(x)
$$

Then $f$ is well-defined and smooth on each simplex, so that $f \in A_{c}^{0}(X)$. But $f$ is not a smooth function of finitely many $t_{i}$ in any neighborhood of 0 in $X_{w}$, so that $f \notin C^{\infty}\left(X_{w}\right)=A^{0}\left(X_{w}\right)$. Now pick $g \in C^{\infty}(\mathbf{R})$ with $g^{-1}(0)=(-\infty, 0)$. Define $h: X \rightarrow \mathbf{R}$ by

$$
h(x)=\sum_{n=1}^{\infty} g\left(t_{n}-1 / n\right) .
$$

Then $h \in C^{\infty}\left(X_{w}\right)=A^{0}\left(X_{w}\right)$, since $h$ is a locally finite sum on $X_{w}$. On the other hand, $h \notin C^{\infty}\left(X_{s}\right)=A^{0}\left(X_{s}\right)$ since $h$ is not a smooth function of finitely many $t_{i}$ on any strong neighborhood of 0 .

\section{Classifying spaces and geometric realizations}

If $C$ is a topological groupoid (= topological category with inverses), then its Milnor-Buffet-Lor classifying space [5] BC is defined to be the space whose points are collections $\left\langle\left\{t_{i}\right\},\left\{c_{i j}\right\}\right\rangle(i, j \in \mathbf{N})$ satisfying

(1) $\left\{t_{i}\right\}$ is a point of the infinite Euclidean simplex $\Delta^{\infty}$ (i.e., $t_{i} \geqslant 0$, all but finitely many $t_{i}$ are zero, and $\Sigma t_{i}=1$ ),

(2) $c_{i j} \in C$ is well-defined only when $t_{i} t_{j} \neq 0$,

(3) $c_{i j} c_{j k}=c_{i k}$ when $t_{i} t_{j} t_{k} \neq 0$.

Remark. This is not the same as Milnor's and Buffet-Lor's original definition of $B C$ as $E C / C$, but is equivalent to it; see [32].

The strong topology $B_{s} C$ on $B C$ is defined to be the coarsest topology 
making the functions $t_{i}: B C \rightarrow \mathbf{R}$ and $c_{i j}:\left\{t_{i} t_{j} \neq 0\right\} \rightarrow C$ continuous. The weak topology $B_{w} C$ on $B C$ is defined as follows. Let $N C$ be the nerve [26] of $C$; this is a semi-simplicial space with

$$
\begin{aligned}
N C[0]= & \text { objects of } C\}, \\
N C[n]= & \left\{\text { composable } n \text {-tuples }\left(c_{1}, \cdots, c_{n}\right) \text { of morphisms in } C\right\} \\
& \text { (i.e., } c_{1} \circ c_{2} \circ \cdots \circ c_{n} \text { is defined), }
\end{aligned}
$$

and with face maps

$$
\partial_{i}: N C[n] \rightarrow N C[n-1], \quad i=0, \cdots, n,
$$

defined by omitting or composing morphisms (see [26], [1]). Now if $S(\sigma)$ is the geometric $n$-simplex of $\Delta^{\infty}$ with vertices $i_{0}<i_{l}<\cdots<i_{n} \in \sigma$, then $S(\sigma) \times$ $N C[n]$ maps to $B C$ by

$$
\left(\left(t_{i_{0}}^{\prime}, \cdots, t_{i_{n}}^{\prime}\right),\left(c_{1}^{\prime}, \cdots, c_{n}^{\prime}\right)\right) \rightarrow\left\langle\left\{t_{i}\right\},\left\{c_{i j}\right\}\right\rangle
$$

where

$$
\begin{gathered}
t_{i}= \begin{cases}t_{i_{j}}^{\prime} & \text { if } i=i_{j}, \\
0 & \text { if } i \neq i_{0}, \cdots, i_{n},\end{cases} \\
c_{i j}=\left\{\begin{array}{l}
c_{k+1}^{\prime} \circ c_{k+2}^{\prime} \circ \cdots \circ c_{m}^{\prime} \text { if } i=i_{k} j=i_{m} \text { with } k<m, \\
c_{j i}^{-1} \text { if } i=i_{k}, j=i_{m} \text { with } k>m, \\
\text { left identity of } c_{1} \text { if } i=j=i_{0}, \\
\text { right identity of } c_{k} \text { if } i=j=i_{k} \text { with } k=1, \cdots, n .
\end{array}\right.
\end{gathered}
$$

These maps induce an isomorphism of sets

$$
\underset{n, \sigma=\sigma_{n}}{\amalg} S(\sigma) \times N C[n] / \sim B C,
$$

where $\sim$ is identification via the face maps:

$$
(t, c) \in S(\sigma) \times N C[n] \sim\left(t, \partial_{j} c\right) \in S\left(\partial_{j} \sigma\right) \times N C[n-1] .
$$

Here $t \in S\left(\partial_{j} \sigma\right) \subset S(\sigma) \subset \Delta^{\infty}, c \in N C[n]$, and $\partial_{j} \sigma$ means $\sigma$ with $i_{j}$ omitted. The weak topology $B_{w} C$ is defined by putting the quotient topology on $B C$ with respect to this map.

More generally, if $X$ is any semi-simplicial space, its unwound geometric realization $\mu(X)$ (called the Milnor geometric realization by tom Dieck [35]; see also [23, Appendix A]), is defined by

$$
\mu(X)=\amalg_{n, \sigma=\sigma_{n}} S(\sigma) \times X[n] / \sim,
$$

where

$$
(t, x) \sim\left(t, \partial_{i} x\right),\left(t \in S\left(\partial_{i} \sigma\right) \subset S(\sigma), x \in X[n]\right)
$$


The weak topology $\mu_{w}(X)$ on $\mu(X)$ is defined to be the quotient topology under the identification map, which we denote by $\pi$. For example, $\mu_{w}(N C)=$ $B_{w} C$. It is also possible to define a strong topology $\mu_{s}(X)$ on $\mu(X)$ in such a way that $\mu_{s}(N C)=B_{s} C$. This works as follows. Observe first that the barycentric coordinates $t_{i}$ of $\Delta^{\infty}$ pull back to global functions (also denoted $t_{i}$ ) on $\mu(X)$. Let $U_{i}=t_{i}^{-1}(0,1] \subset \mu(X)$; the collection $\left\{U_{i}\right\}$ is called the canonical open cover of $\mu(X)$. For each $n$ and each abstract $n$-simplex $\sigma$ of $\Delta^{\infty}$, let $U_{\sigma}=\bigcap_{i \in \sigma} U_{i}$, and let a map

$$
j_{\sigma}: U_{\sigma} \rightarrow X[n]
$$

be defined by

$$
j_{\sigma} \mid S(\tau) \times X[p]: \pi(t, x) \mapsto f_{\sigma \tau}(x),
$$

where $p \geqslant n, \tau$ is an abstract $p$-simplex of $\Delta^{\infty}$ containing $\sigma, t \in S(\tau)$, and $f_{\sigma \tau}$ : $X[p] \rightarrow X[n]$ is the map corresponding to the inclusion $\sigma \subset \tau$ of ordered sets in the semi-simplicial structure of $X$. We define the strong topology $\mu_{s}(X)$ on $\mu(X)$ to be the coarsest topology making all the maps $t_{n}$ and $j_{\sigma}$ continuous.

Example. If $X=N C$ and $\sigma=\left(i_{0}, \cdots, i_{n}\right)$, then

$$
j_{\sigma}\left(\left\{t_{i}\right\},\left\{c_{i j}\right\}\right)=\left(c_{i_{0} i_{1}}, c_{i_{1} i_{2}}, \cdots, c_{i_{n-1} i_{n}}\right) \in N C[n] .
$$

Clearly the topologies $\mu_{s}(N C)$ and $B_{s} C$ are the same.

We now discuss the de Rham cohomology of $\mu(X)$, and note that $B C$ is included as a special case.

Definition. Let $X$ be a semi-simplicial object in the category of differentiable spaces. Then $\mu_{w}(X)$ (resp. $\mu_{s}(X)$ ) is given the smallest differentiable space structure which makes the maps $t_{i}: X \rightarrow \mathbf{R}$ and $j_{\sigma}: U_{\sigma} \rightarrow X[n]$ smooth. (Note that these maps are continuous on both $\mu_{w}(X)$ and $\mu_{s}(X)$.) Explicitly, the smooth functions on $\mu(X)$ are $t_{i}, g \circ j_{\sigma}$ (where $g \in C^{\infty}(U), U$ open in $X[n]$ ), and all functions which locally (on $\mu_{w}(X)$, resp. $\mu_{s}(X)$ ) are smooth functions of these.

The de Rham cohomology of $\mu(X)$ can now be studied by techniques similar to those used on simplicial complexes in the preceding section.

Theorem 8.1. Let $X$ be a semi-simplicial object in the category of differentiable spaces. Then id: $\mu_{w}(X) \rightarrow \mu_{s}(X)$ is a smooth homotopy equivalence.

Proof. Let $\left\{u_{i}\right\}$ be a smooth partition of unity subordinate to the canonical open cover of $\Delta^{\infty}=\mu(0)$ (see $\left.\S 6\right)$. Define

$$
\begin{gathered}
u: \mu(X) \rightarrow \mu(X), \\
u(\pi(t, x))=\pi\left(\left(u_{i}(t)\right), x\right),
\end{gathered}
$$

where $t \in S(\sigma) \subset \Delta^{\infty}, \sigma$ is an $n$-simplex, $\left(u_{i}(t)\right) \in S(\sigma)$, and $x \in X[n]$. The function $u$ is continuous as a map from $\mu_{s}(X)$ to $\mu_{w}(X)$, for if $t \in \operatorname{int}(S(\sigma)$ ), 
then in a neighborhood $W$ of $\pi(t, x) \in \mu_{s}(X)$ we have $u_{j}=0$ for all $j \notin \sigma$ (here we use the fact that $\left\{u_{j}\right\}$ is actually subordinate to the canonical open cover) and $t_{i}>0$ for all $i \in \sigma$, so that $u \mid W$ factors through $S(\sigma) \times X[n]$ via the map $\left[\left(u_{i_{0}}, \cdots, u_{i_{n}}\right), j_{\sigma}\right]$, where $\left(i_{0}, \cdots, i_{n}\right)=\sigma$. Furthermore, $u$ is smooth, since $t_{i} \circ u=u_{i}$, while $j_{\sigma} \circ u=j_{\sigma}$. The linear homotopy $h: \mu(X) \times I$ $\rightarrow \mu(X)$ from the identity to $u$ defines smooth morphisms $h: \mu_{s}(X) \times I \rightarrow$ $\mu_{s}(X)$ and $h: \mu_{w}(X) \times I \rightarrow \mu_{w}(X)$ analogous to those of Theorem 7.1.

Lemma 8.2. The maps $j_{\sigma}: U_{\sigma} \rightarrow X[n]\left(U_{\sigma} \subset \mu_{w}(X)\right)$ are smooth homotopy equivalences.

Proof. The fact that $j_{\sigma}$ is a continuous homotopy equivalence was proved in [23, p. 125] (using the weak topology, but the maps there are continuous in the strong topology, too). The homotopy inverse is the inclusion

$$
i_{\sigma}: X[n] \rightarrow\left\{b_{\sigma}\right\} \times X[n] \subset S(\sigma) \times X[n] \stackrel{\pi}{\rightarrow} \mu(X),
$$

where $b_{\sigma}$ is the barycenter of $S(\sigma)$, and we use the linear homotopy $H_{\sigma}$ : $U_{\sigma} \times I \rightarrow U_{\sigma}$ from id to $i_{\sigma} j_{\sigma}$ where the map $j_{\sigma} i_{\sigma}$ is the identity. Now as in the proof of Theorem $7.2 H_{\sigma}$ pulls back $t_{i}$ to a smooth function, while for any simplex $\rho$, the map $j_{\rho} \circ H_{\sigma}$ equals $j_{\rho}$ wherever it is defined. It follows that $H_{\sigma}$ is smooth.

Theorem 8.3. For any semi-simplicial differentiable space $X$, the de Rham cohomology of $\mu(X)$ (in either topology) can be computed by

$$
H_{D R}(\mu(X))=H_{D}\left(A^{*} X_{*}\right),
$$

where $A^{*} X_{*}$ is the double complex $\bigoplus_{p, q} A^{q} X_{p}$ with de Rham coboundary $d$ : $A^{q} \rightarrow A^{q+1}$, semi-simplicial coboundary

$$
\delta=\Sigma(-1)^{i} A^{q}\left(\partial_{i}\right)=A^{q} X_{p} \rightarrow A^{q} X_{p+1},
$$

and total coboundary $D=d+(-1)^{q} \delta$. This isomorphism is induced by cochain homomorphisms

$$
A^{*}(\mu(X)) \rightarrow A^{*} \mathscr{Q}_{*} \leftarrow A^{*} X_{*},
$$

where $\mathcal{Q}$ is the canonical open cover of $\mu(X)$.

Proof. Since $\mathcal{U}$ admits a subordinate smooth partition of unity (pulled back from $\Delta^{\infty}$ ), Theorem 5.4 implies that

$$
H_{D R}(\mu(X))=H_{D}\left(A^{*} \mathscr{U}_{*}\right) \text {. }
$$

Now the maps $j_{\sigma}: U_{\sigma} \rightarrow X_{p}\left(\sigma=\sigma_{p}\right.$, a $p$-simplex) commute with the face maps $\partial_{i}: U_{\sigma} \subset \mathcal{Q}_{\partial_{i} \sigma}$ and $\partial_{i}: X_{p} \rightarrow X_{p-1}$, and therefore induce a homomorphism of double complexes

$$
j^{*}: A^{*} X_{*} \rightarrow A^{*} \mathscr{U}_{*}
$$


Filtering by $p$ and computing spectral sequences, we get

$$
j^{*}: H_{D R}^{*}\left(X_{p}\right) \rightarrow H_{D R}^{*}\left(\mathcal{U}_{p}\right)=\prod_{\sigma=\sigma_{p}} H_{D R}^{*}\left(U_{\sigma}\right)=\prod_{\sigma=\sigma_{p}} H_{D R}^{*}\left(X_{p}\right)
$$

at the $E_{1}$ level. But the last term is just the semi-simplicial unwinding of the co-semi-simplicial module $p \rightarrow H_{D R}^{*}\left(X_{p}\right)$ (see [23, Appendix $A$ ] and also $\S 9$ below). Since unwinding does not change $\delta$-homology [23, p. 118], it follows that $j^{*}$ induces an isomorphism of $E_{2}$ terms. Since $j^{*}$ was induced by a cochain homomorphism, it follows that it induces isomorphism in total (D-) cohomology.

Corollary 8.4. Let $X$ be a semi-simplicial differentiable space. If the de Rham isomorphism $H_{D R}=H_{s m}=H_{\text {sing }}$ holds on each $X_{p}$, then it holds on $\mu_{s}(X)$ and on $\mu_{w}(X)$.

Proof. It is known [23], [3] that $H_{\text {sing }}(\mu(X))=H_{D}\left(S^{*}\left(X_{*}\right)\right)$; in fact, this follows from Theorem 5.3 using an argument similar to that of Theorem 8.3. A similar proof shows that $H_{s m}(\mu(X))=H_{D}\left(S_{s m}^{*}\left(X_{*}\right)\right)$. Inspection shows that

$$
\begin{array}{ccccc}
H_{D R}(\mu(X)) & \rightarrow & H_{s m}(\mu(X)) & \leftarrow & H_{\text {sing }}(\mu(X)) \\
\downarrow \approx & & \downarrow \approx & & \downarrow \approx \\
H_{D}\left(A^{*}\left(X_{*}\right)\right) & \rightarrow & H_{D}\left(S_{s m}^{*}\left(X_{*}\right)\right) & \leftarrow & H_{D}\left(S^{*}\left(X_{*}\right)\right)
\end{array}
$$

commutes. Filtration of the three double complexes leads to isomorphic $E_{1}$ terms, by hypothesis, hence the maps in the diagram are all isomorphisms. q.e.d.

We observe that in proving Theorem 8.3 we used the Čech-de Rham complex of the canonical open cover of $\mu(X)$, which always admits a smooth partition of unity no matter what $X$ is. For some applications, however, we will need to know when the sheaf $C^{\infty} \mu(X)$ is fine. The following theorem gives a convenient sufficient condition.

Theorem 8.5. Let $X$ be a semi-simplicial differentiable space such that each $X_{n}$ has a metrizable topology and admits smooth partitions of unity (subordinate to any open cover). Then $\mu_{s}(X)$ is metrizable and admits smooth partitions of unity, and the same holds for any subspace of $\mu_{s}(X)$. In particular, the sheaf $\mathrm{C}^{\infty} \mu_{s}(X)$ is fine, as is its restriction to any subspace of $\mu_{s}(X)$.

Proof. By Theorem 6.1, for each $n$ there exist a set $A_{n}$ and a smooth embedding $f_{n}: X_{n} \rightarrow c_{0}\left(A_{n}\right) \subset \mathbf{R}^{A_{n}}$. Let $\left\{U_{i}\right\}$ be the canonical open cover of $\mu(X)$. For each $n$-simplex $\sigma=\left(i_{0}, \cdots, i_{n}\right)$ let $j_{\sigma}: U_{\sigma} \rightarrow X_{n}$ be as before, and let $t_{\sigma}=t_{i_{0}} t_{i_{1}} \cdots t_{i_{n}}: \mu(X) \rightarrow \mathbf{R}$. For each $k \in \mathbf{N}$ define a smooth function $g_{k}$ : $\mathbf{R} \rightarrow[0,1]$ with $g_{k}^{-1}(0,1]=\left(2^{-(k+1)}, 2^{-(k-1)}\right)$. For each $k, n \in \mathbf{N}$ and $n$-simplex 
$\sigma$, define

$$
\begin{gathered}
f_{k n \sigma}: \mu_{s}(X) \rightarrow c_{0}\left(A_{n}\right), \\
f_{k n \sigma}(x)= \begin{cases}g_{k}\left(t_{\sigma}(x)\right) \cdot f_{n}\left(j_{\sigma}(x)\right), & x \in U_{\sigma} \\
0, & t_{\sigma}(x)<w^{-(k+1)}\end{cases}
\end{gathered}
$$

Each $f_{k n \sigma}$ is a smooth morphism, and for each $x \in \mu(X)$ only finitely many $f_{k n \sigma}(x)$ are nonzero. Now let $A=\mathrm{N} \cup \cup_{k, n, \sigma} A_{n}$. Define a map

$$
F: \mu_{s}(X) \rightarrow \mathbf{R}^{A}
$$

by taking the direct product of the maps $\left(t_{0}, t_{1}, \cdots\right): \mu(X) \rightarrow \Delta^{\infty} \subset c_{0}(\mathbf{N}) \subset$ $\mathbf{R}^{\mathbf{N}}$ and the maps $f_{n k \sigma}$. One sees easily that $F$ factors through $c_{0}(A)$ and that $F$ is smooth. Furthermore, $F$ is an embedding; this follows from the fact that locally we can always recover the map $j_{\sigma}$ as $f_{n}^{-1}\left(f_{k n \sigma} / g_{k} \circ t_{\sigma}\right)$ by choosing $k$ suitably. A second application of Theorem 6.1 (together with Remark 1 after it) now shows that $\mu_{s}(X)$ is metrizable and admits smooth partitions of unity. Theorem 6.5 shows that subspaces of $\mu_{s}(X)$ have the same properties.

Examples. $B_{s} G$ and $B_{s} J_{q}$ admit smooth partitions of unity subordinate to any open cover, but $B \Gamma_{q}$ does not. However, Theorem 8.3 is valid for all three spaces.

\section{Comparison of different de Rham theories on geometric realizations}

Let $X$ be a semi-simplicial manifold. There are four cochain complexes which we can use to compute $H_{D R}^{*}(\mu(X))$ (which equals $H_{\text {sing }}^{*}(\mu(X)$ ) if each $X[n]$ satisfies a de Rham isomorphism):

1. The total complex of the double complex $A^{*} X_{*}$ studied by Bott, Shulman and Stasheff [3] (see above, §4).

2. The complex $A^{*}(\|X\|)$ of J. Dupont [14] and C. Watkiss [37], in which an $n$-form $\phi$ is defined as a sequence of $n$-forms $\phi^{(p)} \in A^{n}\left(\Delta^{p} \times X\right)$ satisfying the compatibility conditions

$$
\left(\partial_{i} \times \mathrm{id}\right)^{*} \phi^{(p)}=\left(\mathrm{id} \times \partial_{i}\right)^{*} \phi^{(p-1)}
$$

on $\Delta^{p-1} \times X_{p}$ for all $i=0, \cdots, p$ and all $p=1,2, \cdots$, where $\partial_{i}: \Delta^{p-1} \rightarrow$ $\Delta^{p}$ and $\partial_{i}: X_{p} \rightarrow X_{p-1}$ are face maps.

3. The complex $A^{*}\left(\mu_{w}(x)\right)$ defined in $\S 8$.

4. The complex $A^{*}\left(\mu_{s}(X)\right)$ of $\S 8$.

In this section we shall compare these four complexes, as well as two more complexes obtained by "unwinding" complexes 1 and 2 . We will see that the six complexes are chain homotopy equivalent (c.h.e.), but are not isomorphic.

We first recall the definition of semi-simplicial unwinding [23, Appendix A]. Let $Z_{n}$ be the set of strictly increasing $(n+1)$-tuples $\sigma=\left(i_{0}, \cdots, i_{n}\right)$ of 
nonnegative integers. One defines face maps $\partial_{j}: Z_{n} \rightarrow Z_{n-1}$ by $\partial_{j}\left(i_{0}, \cdots, i_{j}, \cdots, i_{n}\right)=\left(i_{0}, \cdots, \hat{i_{j}}, \cdots, i_{n}\right), j=0, \cdots, n$. If $M$ is a semisimplicial module, then the unwinding $M \times Z$ of $M$ is the facial module (like a semi-simplicial module but with only face maps defined) $n \mapsto \bigoplus_{\sigma \in Z_{n}} M_{n}$ with the obvious face maps. Dually, if $M$ is a co-semi-simplicial module, then its unwinding $M \times Z$ is the co-facial module $n \mapsto \Pi_{\sigma \in Z_{n}} M_{n}$.

Theorem 9.1. [23, p. 118]. Unwinding does not change the homology of a semi-simplicial or co-semi-simplicial module under the boundary map $\delta=$ $\Sigma(-1)^{i} \partial_{i}$. More exactly, if $M$ is a semi-simplicial (resp. co-semi-simplicial) module, then the surjection $M \times Z \rightarrow M$ (resp. injection $M \rightarrow M \times Z$ ) is a chain homotopy equivalence.

(Remark. C. Watkiss [37] proved this independently for co-semi-simplicial modules. He uses "simplicial" to mean simplicial or semi-simplicial in our terminology, while he reserves "semi-simplicial" for what we call "facial".)

Similarly, if $X$ is a semi-simplicial space, then its unwinding $X \times Z$ is the facial space $n \mapsto X_{n} \times Z_{n}$. If $\|X\|$ is the usual unnormalized geometric realization of $X$, defined by

$$
\|X\|=\amalg_{n} \Delta^{n} \times X_{n} / \sim, \text { where }\left(t, \partial_{i}, x\right) \sim\left(\partial_{i} t, x\right), t \in \Delta^{n-1}, x \in X_{n},
$$

then $\|X \times Z\|=\mu_{w}(X)$. (This makes sense even though $X \times Z$ is not a semi-simplicial space, the degeneracy maps not being defined).

Now the Dupont-Watkiss construction makes sense on $X \times Z$ (see Watkiss [37]), so we can speak of $A^{*}(\|X \times Z\|)$, which we can think of as compatible collections of forms on the $S(\sigma) \times X_{p}$, where $S(\sigma)$ is a (nondegenerate) geometric $p$-simplex of $\Delta^{\infty}$.

Finally, we let $A^{*} X_{*} \times Z$ be the algebraic unwinding of the co-semi-simplicial algebra $A^{*} X_{*}$ of Bott, Shulman, and Stasheff.

In [14], Dupont showed that integration of forms over $\Delta^{p}$ (which lowers the degree of a form by $p$ ) defines a chain homotopy equivalence (c.h.e.).

$$
f_{1}: A^{*}(\|X\|) \rightarrow A^{*} X_{*}
$$

The same procedure defines a map

$$
f_{2}: A^{*}(\|X \times Z\|) \rightarrow A^{*} X_{*} \times Z .
$$

Dupont's proof for $f_{1}$ also shows that $f_{2}$ is a c.h.e. (see also Watkiss [37]). On the other hand, the injection

$$
f_{3}: A^{*} X_{*} \rightarrow A^{*} X_{*} \times Z
$$

is a c.h.e. by Theorem 9.1. It follows that the projection $\|X \times Z\| \rightarrow\|X\|$ induces a c.h.e.

$$
f_{4}: A^{*}(\|X\|) \rightarrow A^{*}(\|X \times Z\|)
$$


Now Theorem 8.3 and its proof show that $A^{*}\left(\mu_{w}(X)\right)$ and $A^{*}\left(\mu_{s}(X)\right)$ are chain homotopy equivalent to $A^{*} X_{*}$. Thus we see that all six complexes which we are discussing are chain homotopy equivalent.

We now focus our attention on the exact relationship between our complexes $A^{*}\left(\mu_{w}(x)\right)$ and $A^{*}\left(\mu_{s}(X)\right)$ and the unwound version $A^{*}(\|X \times Z\|)$ of Dupont and Watkiss' complex. The quotient map

$$
\pi: \amalg_{n} \Delta^{n} \times Z_{n} \times X_{n} \rightarrow \mu_{w}(X),
$$

regarded as a morphism of differentiable spaces, pulls back a form on $\mu_{w}(X)$ to a compatible collection of forms on $\|X \times Z\|$, and hence defines a map

$$
h: A^{*}\left(\mu_{w}(x)\right) \rightarrow A^{*}(\|X \times Z\|) \text {. }
$$

The identity map

id: $\mu_{w}(X) \rightarrow \mu_{s}(X)$ induces a map $g: A^{*}\left(\mu_{s}(X)\right) \rightarrow A^{*}\left(\mu_{w}(X)\right)$,

whose chain homotopy inverse is induced by the smooth map $u: \mu_{s}(X) \rightarrow$ $\mu_{w}(X)$ which was defined in the proof of Theorem 8.1 using a smooth partition of unity. The sequence

$$
A^{*}\left(\mu_{s}(X)\right) \stackrel{g}{\rightarrow} A^{*}\left(\mu_{w}(X)\right) \stackrel{h}{\rightarrow} A^{*}(\|X \times Z\|)
$$

is superficially analogous to the sequence

$$
A^{*}\left(Y_{s}\right) \rightarrow A^{*}\left(Y_{w}\right) \rightarrow A_{c}^{*} Y
$$

studied in $\S 7$, where $Y$ is a simplicial complex in the strong or weak topology, and $A_{c}^{*}(Y)$ is the (Whitney) complex of compatible collections of forms on its simplices. We shall imitate our comparison of the terms in the latter sequence to study the former sequence. A major difference between the two cases is that while the strong and weak topologies on a simplicial complex agree when restricted to finite subcomplexes, this is in general not the case for geometric realizations $\mu(X)$, where by a finite subcomplex of $\mu(X)$ we mean that part of $\mu(X)$ lying over a finite subcomplex of $\Delta^{\infty}$. Nonetheless, we have

Theorem 9.2. The maps $A^{*}\left(\mu_{s}(x)\right) \stackrel{g}{\rightarrow} A^{*}\left(\mu_{w}(x)\right) \stackrel{h}{\rightarrow} A^{*}(\|X \times Z\|)$ are strict inclusions. In general, the three cochain complexes disagree even when restricted to a finite subcomplex of $\mu(X)$. They are, however, chain homotopy equivalences, with chain homotopy inverses induced by the map $u: \mu_{s}(X) \rightarrow \mu_{w}(X)$ of Theorem 8.1.

The proof will be broken up into a sequence of lemmas and discussions.

Lemma 9.3. The map $g: A^{*}\left(\mu_{s}(X)\right) \rightarrow A^{*}\left(\mu_{w}(X)\right)$ is injective for any semi-simplicial differentiable space $X$.

Proof. Let $\eta \in$ ker $g$ and suppose $\eta \neq 0$. As in the proof of Lemma 7.4 we can construct a plot $\phi: E \rightarrow \mu_{s}(X)$ such that $\phi^{*} \eta$ is nowhere 0 , and by 
applying the Baire category theorem we can restrict $E$ so that $\phi(E)$ lies over a finite subcomplex of $\Delta^{\infty}$. This in itself does not guarantee that $\phi$ factors through $\mu_{w}(X)$, but if we restrict $E$ further so that $\phi(E)$ lies over the interior of some simplex of $\Delta^{\infty}$, then $\phi$ does factor through $\mu_{w}(X)$, so that $\phi^{*} \eta=0$. This contradiction completes the proof.

Lemma 9.4. The map $h: A^{*}\left(\mu_{w}(X)\right) \rightarrow A^{*}(\|X \times Z\|)$ is injective for any semi-simplicial differentiable space $X$.

Proof. Imitate the proofs of Lemma 9.3 and Lemma 7.5.

Lemma 9.5. The inclusions $A^{*}\left(\mu_{s}(X)\right) \subset A^{*}\left(\mu_{w}(X)\right) \subset A^{*}(\|X \times Z\|)$ are always strict inclusions.

Proof. If we replace $X$ by the trivial semi-simplicial space $P$ with $P_{n}$ a single point for each $n$, then $\mu(P)=\Delta^{\infty}$, and our inclusions reduce to $A^{*}\left(\Delta_{s}^{\infty}\right) \subset A^{*}\left(\Delta_{w}^{\infty}\right) \subset A_{c}^{*}\left(\Delta^{\infty}\right)$, which are strict inclusions by Theorem 7.3 since $\Delta^{\infty}$ is not locally finite. The functions exhibited in the proof of Lemma 7.8 to prove this fact pull back to $\mu(X)$ and provide examples to prove the present lemma. q.e.d.

Actually, a stronger statement is true. If we restrict the three cochain complexes to a finite subcomplex of $\mu(X)$, they are still not isomorphic, in general (unlike the case of simplicial complexes). This phenomenon can be illustrated by considering the semi-simplicial space $X=N R$, where $R$ is the real line regarded as a topological group under addition. The portion of $\mu(X)=B R$ lying over $\Delta^{1} \subset \Delta^{\infty}$ is then the suspension of $R$, namely

$$
S R=I \times R /[(0, r) \sim(0, s) ;(1, r) \sim(1, s), \text { for all } r, s \in R],
$$

where $I=[0,1]$, toplogized in either the weak topology $S R_{w}$ as a quotient of $I \times R$ or the strong topology $S R_{s}$, which is the coarsest topology in which the coordinate projections

$$
\begin{aligned}
& t: S R \rightarrow I, \\
& r: S R-O^{*}-1^{*} \rightarrow R
\end{aligned}
$$

are continuous, where $0^{*}=$ image of $(0, r)$ (any $\left.r \in R\right), 1^{*}=$ image of $(1, r)$. Any smooth function $f: I \times R \rightarrow R$ which is constant on $\{0\} \times R$ and on $\{1\} \times R$ defines a Dupont-Watkiss-smooth function $f_{0}$ on $S R$. In order for $f_{0}$ to lie in $C^{\infty}\left(S R_{w}\right)$, however, $f$ must be a (smooth) function of $t$ alone in some neighborhood of $\{0\} \times R$ and $\{1\} \times R \subset I \times R$, while for $f_{0}$ to lie in $C^{\infty}\left(S R_{s}\right), f$ must be a function of $t$ alone on some such neighborhood of the special form $\{t<\varepsilon$ or $t>1-\varepsilon\}$. For example, the function $t r$ restricted to $\left\{t<\frac{1}{2}\right\} \subset S R$ is Dupont-Watkiss smooth but not weak-smooth, while if $g \in C^{\infty}(\mathbf{R})$ has support $=[0, \infty)$ then the function $f: S R-1^{*} \rightarrow \mathbf{R}$ defined 
by

$$
f(t, r)= \begin{cases}g(r-1 / t), & t>0 \\ 0, & t=0\end{cases}
$$

is weak-smooth but not strong-smooth.

This example can also be used to illustrate the problem with trying to pull back Watkiss-Dupont forms on $\|X\|$ or $\|X \times Z\|$ to a manifold $M$ via a smooth map $f: M \rightarrow\|X\|$ (using some reasonable definition of what it means for $f$ to be smooth) or $f: M \rightarrow\|X \times Z\|=\mu(X)$ (using our previous definition that $f$ be a smooth morphism). If such a map happens to factor locally through $\amalg_{n} \Delta^{n} \times X_{n}$ (resp. $\amalg_{n} \Delta^{n} \times X_{n} \times Z_{n}$ ), then any Watkiss-Dupont form $\eta \in A^{*}(\|X\|)$ or $A^{*}(\|X \times Z\|)$ can be pulled back locally by $f$. The local pullbacks will agree because they are pulled back from a compatible collection of forms. Therefore a global pull-back $f^{*}(\eta)$ on $M$ is defined in this case. In the general case, however, one can have a smooth morphism $f$ : $M \rightarrow \mu_{w}(X)$ which does not factor locally in this way, and then one may not be able to pull back Watkiss-Dupont forms. For example, consider the map

$$
\begin{gathered}
f:(-0.5,0.5) \rightarrow S R_{w}, \\
f(x)= \begin{cases}\left(x^{2}, 4\right) & x>0 \\
0^{*}, & x=0 \\
\left(x^{2}, 3\right), & x<0\end{cases}
\end{gathered}
$$

Now $f$ is continuous, and it is also smooth because $t \circ f(x)=x^{2} \in$ $C^{\infty}(-0.5,0.5)$, while $r \circ f$ is smooth on $(-0.5,0.5)-\{0\}$. (Recall that $r$ is not defined on $0^{*} \in S R$ ). The function $\operatorname{tr}$ is Watkiss-Dupont-smooth on $\{t<$ $1 / 2\} \subset S R$, but its pullback (tr) $\circ f$ equals $4 x^{2}$ when $x>0$ and $3 x^{2}$ when $x<0$, and is therefore not smooth.

The same map $u: \mu_{s}(X) \rightarrow \mu_{w}(X)$ which is a smooth homotopy inverse of id: $\mu_{w}(X) \rightarrow \mu_{s}(X)$ (see Theorem 8.1) can be used to construct an explicit chain homotopy inverse

$$
k: A^{*}(\|X \times Z\|) \rightarrow A^{*}\left(\mu_{s}(X)\right)
$$

to the map

$$
h g: A^{*}\left(\mu_{s}(X)\right) \rightarrow A^{*}(\|X \times Z\|) .
$$

To do this, we observe, following the proof of Theorem 8.1, that $u$ factors locally through $S(\sigma) \times X_{n}$, where $S(\sigma)$ is an $n$-simplex of $\Delta^{\infty}$, so that a form in $A^{*}(\|X \times Z\|)$ can be pulled back locally (and hence globally) via $u$ to a form in $A^{*}\left(\mu_{s}(X)\right)$. This defines the map $k$. The linear homotopy $E: \mu_{s}(X) \times$ $I \rightarrow \mu_{s}(X)$, called $h$ in Theorem 8.1, from the identity to $u$ is built from a 
collection of smooth homotopies

$$
\begin{gathered}
E_{\sigma}: S(\sigma) \times X_{n} \times I \rightarrow S(\sigma) \times X_{n}, \\
E_{\sigma}(t, x, s)=\left[\left((1-s) t_{i_{0}}(t)+s u_{i_{0}}(t), \cdots,(1-s) t_{i_{n}}(t)+s u_{i_{n}}(t)\right), x\right],
\end{gathered}
$$

(where $t \in S(\sigma) \subset \Delta^{\infty}, \sigma=\left(i_{0}, \cdots, i_{n}\right)$ ), which are compatible under identifications via face maps. A chain homotopy from the identity map on $A^{*}\left(\mu_{s}(X)\right)$ to $k \circ h g$ is obtained by composing

$$
A^{*}\left(\mu_{s}(X)\right) \stackrel{E^{*}}{\rightarrow} A^{*}\left(\mu_{s}(X) \times I\right) \stackrel{L}{\rightarrow} A^{*-1}\left(\mu_{s}(X)\right),
$$

where $L$ is the usual homotopy operator (see $\S 3$ ). Similarly, a chain homotopy from id to $h \mathrm{~g} \circ k$ is given by $L \circ\left\{E_{\sigma}\right\}^{*}$. To summarize we have

Lemma 9.6. The inclusions

$$
A^{*}\left(\mu_{s}(X)\right) \subset A^{*}\left(\mu_{w}(X)\right) \subset A^{*}(\|X \times Z\|)
$$

are chain homotopy equivalences. $A$ chain homotopy inverse for any of the three inclusions is induced by the smooth map $u: \mu(X) \rightarrow \mu(X)$ defined in the proof of Theorem 8.1.

Intuitive Explanation. The example of the space $S R$ discussed above shows that when restricted to finite subcomplexes of $\mu(X)$, the three de Rham theories $A^{*}\left(\mu_{s}(X)\right), A^{*}\left(\mu_{w}(X)\right)$, and $A^{*}(\|X \times Z\|)$ differ in their behavior near the boundary of each simplex. The first two theories are more controlled near boundaries, and therefore forms in those theories can always be pulled back via smooth morphisms $f: M \rightarrow \mu(X)$. The smooth map $u: \mu(X) \rightarrow \mu(X)$ used in Lemma 9.6 pushes a neighborhood of the boundary of each simplex into the boundary, and therefore "tames" forms near the boundary when it pulls them back.

\section{Applications}

In this section we shall discuss some applications of differentiable spaces. We believe that the potential applications are much more numerous than those presented here, because the differentiable space construction is very general. Indeed, the de Rham theorems of $\$ 5$ can be thought of as giving criteria for selecting a distinguished class of functions on a space $X$ which yields the correct real cohomology of $X$ when plugged into the differentiable space construction.

The applications which we have discovered so far are in the realm of characteristic classes of bundles and of foliations. One such use involves extending smooth and $C^{k}$ cohomology theories on spaces with two topologies to the classifying space for foliations in its germ $\left(B \Gamma_{q}\right)$ and jet $\left(B J_{q}\right)$ topologies. Most of the details of this application have already appeared in [24], so 
we will only sketch the results and fill in the gaps in that account. The history of this problem begins with Bott and Haefliger [2], who defined a singular cohomology theory $H_{c}^{*}\left(X^{\prime} \rightarrow X\right)$ on spaces $X^{\prime}$ which possess a coarser topology $X$, based on singular real-valued cochains on $X^{\prime}$ whose values vary continuously when simplices (of $X^{\prime}$ ) are moved continuously through $X$. They conjectured that $H_{c}^{*}\left(B \Gamma_{q} \rightarrow B J_{q}\right)$ equals $H^{*}\left(W O_{q}\right)$; the latter was already known (by the work of Bott, Haefliger, Kamber, Tondeur, Godbillon, Vey, et al.) as the algebra of potential characteristic classes for foliations which can be constructed from curvatures and connections by exploiting the Bott vanishing phenomenon. In [23] and [24] we studied the properties of $H_{c}$ and of other continuous, smooth, and $C^{k}$ cohomology theories on the category of morphisms of manifolds. In particular, we defined the $C^{k}$ cohomology theory $T_{k m n}^{*}\left(X^{\prime} \rightarrow X\right)=H \Gamma S_{k m n}^{*}\left(X^{\prime} \rightarrow X\right)$, where $\Gamma=$ global sections and $\mathbf{S}_{k m n}^{q}\left(X^{\prime}\right.$ $\rightarrow X)$ is the sheaf on $X$ generated by real-valued cochains on the $C^{n}$ singular $q$-simplices of $X^{\prime}$ which vary in a $C^{k}$ manner when simplices (of $X^{\prime}$ ) are moved through $X$ in a $C^{m}$ manner. In order to extend this theory to $\left(B \Gamma_{q} \rightarrow B J_{q}\right)$ we observed that its definition makes sense in any category of topological spaces on which some notion of $C^{n}$ functions exists for each $n=1,2, \cdots, \infty$. The category of differentiable spaces is ideally suited for this purpose-one defines a function $f: X \rightarrow \mathbf{R}$ to be $C^{n}$ if it is locally a $C^{n}$ function of finitely many smooth functions.

The theory $T_{k m n}$, when restricted to morphisms $\left(X^{\prime} \rightarrow X\right)$ of paracompact manifolds, is invariant under smooth homotopies and satisfies an extended Mayer-Vietoris theorem (analogous to Theorems 5.3 and 5.4) relative to open covers of $X$. Does $T_{k m n}$ have the same properties on the category of morphisms $\left(X^{\prime} \rightarrow X\right)$ of differentiable spaces? To answer this, we first observe that the sheaf $\mathbf{S}_{k m n}^{*}\left(X^{\prime} \rightarrow X\right)$ is a module over $\mathbf{C}^{\infty} X$. If $X$ is paracompact and admits smooth partitions of unity, then $\mathbf{C}^{\infty} X$ is fine, implying that $\mathbf{S}_{k m n}^{*}\left(X^{\prime} \rightarrow\right.$ $X)$ is fine. The proof of smooth homotopy invariance goes through if $\mathrm{S}_{k m n}^{*}\left(X^{\prime} \times I \rightarrow X \times I\right)$ is fine and $X \times I$ paracompact. It follows that $T_{k m n}$ is smooth homotopy invariant on the category of morphisms $\left(X^{\prime} \rightarrow X\right)$ of differentiable spaces such that $X$ (and hence $X \times I$ ) is paracompact and admits smooth partitions of unity. It satisfies the Extended Mayer-Vietoris Theorem on the same category (imitate the proof of Theorem 5.4). If $\left(X^{\prime} \rightarrow X\right)$ is a semi-simplicial object in the category of differentiable spaces, and each $X_{n}$ is metrizable and admits smooth partitions of unity, then $\mu_{s}(X)$, all its subspaces, and all its subspaces crossed with $I$ are metrizable and admit smooth partitions of unity (by Theorems 8.5 and 6.1) so that we can imitate the proof of Theorem 8.3 to obtain the isomorphism

$$
T_{k m n}^{*}\left(\mu_{s}\left(X^{\prime}\right) \rightarrow \mu_{s}(X)\right)=H_{D}\left(\Gamma S_{k m n}^{*}\left(X_{*}^{\prime} \rightarrow X_{*}\right)\right)
$$


In particular,

$$
T_{k m n}^{*}\left(B \Gamma_{q} \rightarrow B J_{q}\right)=H_{D}\left(\Gamma S_{k m n}^{*}\left(N \Gamma_{q}[*] \rightarrow N J_{q}[*]\right)\right) .
$$

Although this last isomorphism shows, a posteriori, that one could have defined $T_{k m n}^{*}\left(B \Gamma_{q} \rightarrow B J_{q}\right)$ without using differentiable spaces, the differentiable space definition makes it easier to pull back classes to manifolds. For example a codimension $q$ foliation on a manifold $M$ gives rise to a smooth cocycle on $M$ with values in $\Gamma_{q}$ which is classified by a smooth morphism $f$ : $M \rightarrow B \Gamma_{q}$ (see $\S 1$, Example 7). A smooth one-parameter family of foliations on $M$ is classified by a commutative diagram

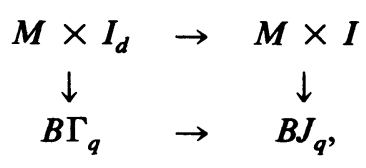

where $I$ is the unit interval, and $I_{d}$ is $I$ with the discrete topology. Such a diagram induces a homomorphism

$$
\begin{aligned}
T_{k m n}^{*}\left(B \Gamma_{q} \rightarrow B J_{q}\right) & \rightarrow T_{k m n}^{*}\left(M \times I_{d} \rightarrow M \times I\right) \\
& =\operatorname{Hom}_{\mathrm{R}}\left(H_{*}(M), C^{k}(I)\right) .
\end{aligned}
$$

In other words, the elements of $T_{k m n}^{*}\left(B \Gamma_{q} \rightarrow B J_{q}\right)$ can be regarded as characteristic classes of foliations which vary in a $C^{k}$ manner as a foliation is varied smoothly, but the author does not know if all $C^{k}$ characteristic classes of foliations can be obtained in this way.

Another cohomology theory on morphisms of manifolds is the smooth theory

$$
T_{D R}^{*}\left(X^{\prime} \rightarrow X\right)=H^{*}\left(\operatorname{image}\left(A^{*} X \rightarrow A^{*} X^{\prime}\right)\right),
$$

(see [23]). The exact same definition works on the category $\mathscr{D}_{2}$ of morphisms of differentiable spaces. The theory $T_{D R}$ is smooth homotopy invariant on the whole category $\mathscr{D}_{2}$; this is proved exactly like Theorem 3.3 , using the fact that the homotopy operator $L: A^{*}(X \times I) \rightarrow A^{*-1}(X)$ is natural in $X . T_{D R}$ satisfies an extended Mayer-Vietoris theorem on $\mathscr{D}_{2}$, but only relative to open covers of $X$ which admit smooth partitions of unity. (The proof of Theorem 5.4 can be adapted to $\mathscr{D}_{2}$ since image $\left(A^{*} X \rightarrow A^{*} X^{\prime}\right)$ is a module over $C^{\infty}(X)$.) These two properties are sufficient as in the proof of Theorem 8.3 to prove that

$$
T_{D R}^{*}\left(\mu\left(X^{\prime}\right) \rightarrow \mu(X)\right)=H_{D}\left(\operatorname{image}\left(A^{*} X_{*} \rightarrow A^{*} X_{*}^{\prime}\right)\right)
$$

for any morphism $\left(X^{\prime} \rightarrow X\right)$ of semi-simplicial differentiable spaces; here $\mu\left(X^{\prime}\right)$ and $\mu(X)$ may be given the weak or the strong topology. In case 


$$
\begin{aligned}
& \left(X^{\prime} \rightarrow X\right)=\left(N \Gamma_{q} \rightarrow N J_{q}\right), \text { we get } \\
& T_{D R}^{*}\left(B \Gamma_{q} \rightarrow B J_{q}\right)=H_{D}\left(\operatorname{image}\left(A^{*} N J_{q}[*] \rightarrow A^{*} N \Gamma_{q}[*]\right)\right),
\end{aligned}
$$

which equals $H^{*}\left(W O_{q}\right)$ by an unpublished result of Bott and Haefliger.

Another use of differentiable spaces is to yield universal formulas for differential forms representing characteristic classes of $G$-bundles, where $G$ is a Lie group. We remark that Dupont [15] and Watkiss [37] have made similar constructions in their de Rham theories, and that Shulman [27] constructed characteristic forms in the double complex $A^{*} N G$. Since $H_{D R}^{*}(B G)=$ $H_{\text {sing }}^{*}(B G)$ (by Corollary 8.4; $B G$ can have the weak or the strong topology here), every real characteristic class for $G$-bundles is represented by differential forms in $A^{*}(B G)$. Furthermore, since a $G$-bundle with smooth transition functions on a manifold $M$ is classified by a smooth morphism $f: M \rightarrow B G$ (\$1, Ex. 7), these characteristic forms on $B G$ can be pulled back to differential forms (not just cohomology classes) on $M$. Since any form $\eta$ on $B G$ can be expressed in terms of the functions $t_{i}$ and $g_{i j}$ and their differentials, it follows that $f^{*} \eta$ will be expressed in terms of the transition functions and a smooth partition of unity subordinate to a trivializing open cover of $\boldsymbol{M}$.

To construct explicit characteristic forms on $B G$, we mimic the Chern-Weil approach using connections and curvatures (see [6]). Let $E G$ be the total space of the universal $G$-bundle over Milnor's $B G$; it is defined by

$$
\begin{aligned}
E G=\left\{\text { collections }\left\langle t,\left(g_{i}\right)\right\rangle\right. \text { such that } \\
\left.\qquad t=\left(t_{i}\right) \in \Delta^{\infty} \text { and } g_{i} \in G \text { is defined when } t_{i} \neq 0\right\}
\end{aligned}
$$

in the strong topology, the coarsest topology making all $t_{i}$ and $g_{i}$ continuous, [21]. ( $E G=\mu(P G)$, where $P G$ is the semi-simplicial manifold

$$
G \leftleftarrows G \times G \cdots
$$

defined by the homogeneous complex of $G$.) The bundle projection $p$ : $E G \rightarrow B G$ is defined as usual by

$$
p:\left\langle t,\left(g_{i}\right)\right\rangle \rightarrow\left\langle t, g_{i j}=g_{i} g_{j}^{-1}\right\rangle .
$$

We make $E G=\mu(P G)$ into a differentiable space in the usual way; this amounts to saying that $f: E G \rightarrow \mathbf{R}$ is smooth it is is locally a smooth function of finitely many of the $t_{i}$ and $g_{i}$. Let $\theta$ be the canonical g-valued 1 -form on $G$ $\left[18\right.$, p. 41]. Then $g_{i}^{*} \theta=\left(\right.$ def.) $\omega_{i}$ is a $g$-valued 1-form on $\left\{t_{i} \neq 0\right\} \subset E G$, and $\omega=\left(\right.$ def.) $\Sigma_{i} u_{i} \omega_{i}$ (where $\left\{u_{i}\right\}$ is a smooth partition of unity on $E G$ (pulled up from $\left.\Delta^{\infty}\right)$ subordinate to the canonical open cover) belongs to $A^{1}(E G ; g)$ and acts like a connection form for the bundle $p: E G \rightarrow B G$, even though $E G$ and 
$B G$ do not have tangent spaces. For example, any smooth $g$-bundle map

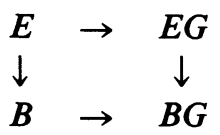

pulls back $\omega$ to an honest connection form. We can define the curvature $\Omega \in A^{2}(E G ; \mathrm{g})$ of $\omega$ by using the structure equation $[18$, p. 78]

$$
\Omega=\text { (def.) } d \omega+\frac{1}{2}[\omega, \omega]=\sum_{i} d \omega^{i} \otimes e_{i}+\frac{1}{2} \sum_{i, j, k} C_{j k}^{i}\left(\omega^{j} \wedge \omega^{k}\right) \otimes e_{i},
$$

where $\left\{e_{i}\right\}$ is a basis of $\mathrm{g}, \omega=\Sigma_{i} \omega^{i} \otimes e_{i}$, and $C_{j k}^{i}$ are the structure constants of $\mathrm{g}$. If $\phi$ is a $\mathrm{g}$-invariant polynomial of degree $k$ on $\mathrm{g}$, then we define $\phi(\Omega) \in A^{2 k}(E G)$ as usual, following Chern-Weil [6].

Theorem 10.1 The form $\phi(\Omega)$ is horzontal, i.e., there exists a form $\psi \in$ $A^{2 k}(B G)$ with $p^{*} \psi=\phi(\Omega)$. The form $\psi$ is unique and closed $(d \psi=0)$.

Proof. Since we do not have tangent vectors on $B G$ and $E G$, we must resort to an indirect proof. We construct a form $\psi$ explicitly and then show that $p^{*} \psi=\phi(\Omega)$. On $\left\{t_{n} \neq 0\right\} \subset B G$, define a local section $s_{n}$ of $p: E G \rightarrow B G$ by

$$
s_{n}:\left\langle t,\left(g_{i j}\right)\right\rangle \mapsto\left\langle t,\left(g_{i}=g_{i n}\right)\right\rangle .
$$

Define

$$
\psi=\Sigma_{n} u_{n} \cdot s_{n}^{*} \phi(\Omega) \in A^{2 k}(B G),
$$

where $\left\{u_{n}\right\}$ is again a smooth partition of unity pulled up from $\Delta^{\infty}$. We claim that $p^{*} \psi=\phi(\Omega)$. To show this it suffices to show that any plot $h: K \rightarrow E G$ pulls back $p^{*} \psi$ and $\phi(\Omega)$ to identical forms in $A^{*}(K)$. But this follows fairly easily from the fact that $h^{*} \phi(\Omega)=\phi\left(h^{*} \Omega\right)$ and that $\phi$ of the curvature form of any $G$-bundle over a manifold is a horizontal form. The other assertions are proved similarly.

Remarks. Theorem 10.1 exhibits a factorization of the usual Chern-Weil homomorphism (at the differential form level) through $A^{*}(B G)$. The proof of Theorem 10.1 not only shows that $\psi$ exists but also gives an explicit formula for it in terms of the functions $u_{i}, g_{i j}$ and their differentials.

The preceding applications should illustrate the usefulness of differentiable spaces in studying real cohomology at the cochain level with differential forms. In future papers we hope to use the differentiable space concept to study other spaces which admit suitable classes of distinguished functions.

\section{References}

[1] R. Bott, Lectures on characteristic classes and foliations, Lecture Notes in Math. Vol. 279, Springer, Berlin, 1972, 1-94. 
[2] - Some remarks on continuous cohomolgoy, Manifolds-Tokyo 1973, University of Tokyo Press, Tokyo, 1975, 161-170.

[3] R. Bott, H, Shulman \& J. Stasheff, De Rham theory for classifying spaces, Advances in Math. 20 (1976) 43-56.

[4] G. Bredon, Sheaf theory, McGraw-Hill, New York, 1967.

[5] J. P. Buffet \& J. C. Lor, Une construction d'un universel pour une classe assez large de r-structures, C. R. Acad. Sci. Paris, Série A, 270 (1970) 640-642.

[6] H. Cartan, Cohomologie réelle d'un espace fibré principal différentiable, Seminaire Henri Cartan, 1949-50, Exp. 19; second ed., 1956.

[7] Theories cohomologiques, Invent. Math. 35 (1976) 261-271.

[8] K. T. Chen, Iterated integrals of differential forms and loop space homology, Ann. of Math. 97 (1973) 217-246.

[9]___ Iterated integrals, fundamental groups and covering spaces, Trans. Amer. Math. Soc. 206 (1975) 83-98.

[10] A. Dold. Partitions of unity in the theory of fibrations, Ann. of Math. 78 (1963) 223-255.

[11] _Lectures on algebraic topology, Springer, Berlin, 1972,

[12] C. H. Dowker, Topology of metric complexes, Amer. J. Math. 74 (1952) 555-577.

[13] J. Dugundji, Topology, Allyn and Bacon, Boston, 1966.

[14] J. L. Dupont, Simplicial De Rham cohomology and characteristic classes of flat bundles, Aarhus Univ. Preprint Series, No. 29, 1974-75, and Topology 15 (1976) 233-245.

[15] M. J. Greenberg, Lectures on algebraic topology, Benjamin, New York, 1967.

[16] A. Haefliger, Homotopy and integrability, Lecture Notes in Math. Vol. 197, Springer, Berlin, 1971, 133-163.

[17] F. Kamber \& P. Tondeur, Semisimplicial Weil algebras and characteristic classes for foliated bundles in Cech cohomology, Proc. Sympos. Pure Math. Vol. 27, Amer. Math. Soc., 1975, 283-294.

[18] S. Kobayashi \& K. Nomizu, Foundations of differential geometry, Vol. 1, Interscience, New York, 1963.

[19] N. H. Kuiper, On the smoothings of triangulated and combinatorial manifolds, Differential and Combinatorial Topology, Princeton University Press, Princeton, 1965, 3-22.

[20] C. D. Marshall, The de Rham cohomology of subcartesian spaces, J. Differential Geometry 10 (1975) 575-588.

[21] J. Milnor, Construction of universal bundles. II, Ann. of Math. 63 (1956) 430-436.

[22] On spaces having the homotopy type of a CW-complex, Trans. Amer. Math. Soc. 90 (1959) 272-280.

[23] M. A. Mostow, Continuous cohomology of spaces with two topologies, Mem. Amer. Math. Soc. No. 175, 1976.

[24] __ Variations, characteristic classes, and the obstruction to mapping smooth to continuous cohomology, Trans.Amer. Math. Soc. 240 (1978) 163-182.

[25] R. S. Palais, The cohomology of Lie rings, Proc. Sympos. Pure Math. Vol. 3, Amer. Math. Soc., 1961, 130-137.

[26] G. Segal, Classifying spaces and spectral sequences, Inst. Hautes Etudes Sci. Publ. Math. No. 34 (1968) 105-112.

[27] H. Shulman, Characteristic classes and foliations, Thesis, Berkeley, 1972.

[28] H. Shulman \& J. Stasheff, De Rham theory for BГ, Proc. Conf. on Foliations, Rio de Janeiro, 1976.

[29] R. Sikorski, Abstract covariant derivative, Colloq. Math. 18 (1967) 251-279.

[30] J. W. Smith, The de Rham theorem for general spaces, Tôhoku Math. J. 18 (1966) 115-137.

[31] E. Spanier, Algebraic topology, McGraw-Hill, New York, 1966.

[32] J. Stasheff, Parallel transport and classification of fibrations, Lecture Notes in Math. Vol. 428, Springer, Berlin, 1974. 
[33] D. Sullivan, Differential forms and the topology of manifolds, Manifolds-Tokyo 1973, University of Tokyo Press, Tokyo, 1975, 37-49.

[34] R. G. Swan, Thom's theory of differential forms on simplicial sets, Topology 14 (1975) 271-273.

[35] T. tom Dieck, On the homotopy type of classifying spaces, Manuscripta Math. 11 (1974) 41-49.

[36] H. Torunczyk, Smooth partitions of unity of some non-separable Banach spaces, Studia Math. 46 (1973), 43-51.

[37] C. Watkiss, Cohomology of principal bundles in semi-simplicial theory, Thesis, University of Toronto, 1975.

[38] A. Weil, Sur les théorèmes de de Rham, Comment. Math. Helv. 26 (1952) 119-145.

[39] J. H. C. Whitehead, Combinatorial homotopy, I. Bull. Amer. Math. Soc. 55 (1949) 213-245.

[40] H. Whitney, Geometric integration theory, Princeton University Press, Princeton, 1957.

UNIVERSITY OF ILLINOIS, URBANA 
